SumitTed to ApJ 2009 JANUARY 20; ACCEPTED 2009 APRIL 13

Preprint typeset using LATEX style emulateapj v. 08/13/06

\title{
ELECTRIC CHARGING OF DUST AGGREGATES AND ITS EFFECT ON DUST COAGULATION IN PROTOPLANETARY DISKS
}

\author{
SATOSHI OKUZUMI \\ Graduate School of Human and Environmental Studies, Kyoto University, Yoshida-nihonmatsu-cho, Sakyo-ku, Kyoto 606-8501, Japan \\ Sumitted to ApJ 2009 January 20; accepted 2009 April 13
}

\begin{abstract}
Mutual sticking of dust aggregates is the first step toward planetesimal formation in protoplanetary disks. In spite that the electric charging of dust particles is well recognized in some contexts, it has been largely ignored in the current modeling of dust coagulation. In this study, we present a general analysis of the dust charge state in protoplanetary disks, and then demonstrate how the electric charging could dramatically change the currently accepted scenario of dust coagulation. First, we describe a new semianalytical method to calculate the dust charge state and gas ionization state self-consistently. This method is far more efficient than previous numerical methods, and provides a general and clear description of the charge state of gas-dust mixture. Second, we apply this analysis to compute the collisional cross section of growing aggregates taking their charging into account. As an illustrative example, we focus on early evolutionary stages where the dust has been thought to grow into fractal $(D \sim 2)$ aggregates with a quasi-monodisperse (i.e., narrow) size distribution. We find that, for a wide range of model parameters, the fractal growth is strongly inhibited by the electric repulsion between colliding aggregates and eventually "freezes out" on its way to the subsequent growth stage involving collisional compression. Strong disk turbulence would help the aggregates to overcome this growth barrier, but then it would cause catastrophic collisional fragmentation in later growth stages. These facts suggest that the combination of electric repulsion and collisional fragmentation would impose a serious limitation on dust growth in protoplanetary disks. We propose a possible scenario of dust evolution after the freeze-out. Finally, we point out that the fractal growth of dust aggregates tends to maintain a low ionization degree and, as a result, a large magnetorotationally stable region in the disk.

Subject headings: dust, extinction — methods: analytical — planetary systems: formation — planetary systems: protoplanetary disks — plasmas
\end{abstract}

\section{INTRODUCTION}

The initial step toward planetesimal formation in protoplanetary disks is the collisional growth of submicron dust grains into macroscopic aggregates. A standard scenario is that dust aggregates grow by mutual sticking, gradually settle to the midplane of the disk, and finally form a dense dust layer. It is still an open issue whether subsequent growth is established by the gravitational instability of the layer or the direct growth of the aggregates. To address this issue, further understanding on earlier evolutionary stages is needed.

It has been recognized that the internal structure of aggregates is a key factor for their growth and settling. Early studies on dust coagulation modeled the aggregates as a compact, nonporous object (e.g., Weidenschilling 1980; Nakagawa et al. 1981). Both numerical simulations and laboratory experiments have revealed, however, that aggregates are not at all compact, but has an open, fluffy structure (for a review, see Meakin 1991; Blum 2004; Dominik et al. 2007). This is particularly true for aggregates formed at an early growth stage where the collisional velocity is too low for colliding aggregates to compress each other. It has been observed in numerical (Kempf et al. 1999) as well as experimental (Wurm \& Blum 1998; Blum et al. 1998, 2000) studies that the outcome is an ensemble of fractal aggregates with the fractal dimension $D \lesssim 2$ and with a quasi-monodisperse (i.e., narrow) mass distribution. This fractal growth typically lasts until the aggregates become centimeter-sized (Suyama et al. 2008). A remarkable dynamical property of these fluffy aggregates is that they keep a strong coupling to ambient gas and

Electronic address: satoshi.okuzumi@ax2.ecs.kyoto-u.ac.jp thus a low drift velocity relative to the gas throughout the evolution. This could be crucial to the formation of very thin dust layer where planetesimals may be formed by gravitational instability.

Dust grains and aggregates are not only the building block of planetesimals but also powerful absorbers of charged particles in the gas disks. It is now widely accepted that turbulence in the disks is attributed to magnetorotational instability (MRI; Balbus \& Hawley 1991). For this mechanism to work, at least a part of the disk needs to be sufficiently ionized for the gas to couple to magnetic fields. Many authors have examined whether protoplanetary disks can be ionized enough to sustain MHD turbulence (Gammie 1996; Glassgold et al. 1997; Sano et al. 2000; Igea \& Glassgold 1999; Ilgner \& Nelson 2006a b. c; Wardle 2007). One of the important findings is that the turbulent region is strongly controlled by the concentration of dust materials since they efficiently remove away ionized particles from ambient gas (Sano et al. 2000; Ilgner \& Nelson 2006a; Wardle 2007).

Although the importance of dust charging is well recognized in the above context, its effect on dust coagulation in protoplanetary disks has been hardly examined. Charging of aggregates causes electrostatic interaction between them, which may significantly increase or decrease the coagulation rates. Recently, a series of studies have suggested that charge-induced dipole interaction might trigger runaway growth of dust aggregates (Ivlev et al. 2002; Konopka et al. 2005). However, these studies considered a situation where the ambient gas is not ionized and the net charge of dust aggregates vanishes identically. In protoplanetary disks, on the contrary, the net charge of dust aggregates does not vanish 
due to the presence of weakly ionized ambient gas, and therefore both dust charging and gas ionization must be taken into account.

This study explores how the electrostatic charging of dust aggregates could be crucial to their coagulation in protoplanetary disks. For this purpose, we have to know in advance how the charge state of aggregates evolves with their growth. This is a complicated problem, since we also have to solve the ionization state of ambient gases self-consistently. Previous studies (Sano et al. 2000; Ilgner \& Nelson 2006a; Wardle 2007) have handled this problem with direct numerical calculations in which dust particles with different charges and sizes are treated as different charged species as well as many species of ions. However, this approach becomes inefficient when one tries to solve this problem and dust growth simultaneously, since the dispersion of charge and size increases as the dust growth. The central strategy taken in this study is to solve this problem as analytically as possible. This approach does not only reduce the computational expense but also provides general insight into the charge state of gas-dust mixture. As a result, we show that all the conditions for ionizationrecombination equilibrium are reduced to a single algebraic equation. Just by solving this equation numerically, we can obtain both of the dust charge state and the gas ionization state analytically. We also confirm that the semianalytical calculations agree very well with direct numerical calculations using the original equations. This semianalytical method will be a powerful tool for the simulations of charged dust coagulation and MRI turbulence.

As an illustrative example, we calculate the collisional cross section of dust aggregates growing in a protoplanetary disk taking into account their electric charging. We focus on early stages of dust evolution where the aggregates has been thought to experience fractal, quasi-monodisperse growth (e.g., Blum 2004; Dominik et al. 2007). For a wide range of model parameters, we find that the effective cross section is quickly suppressed as the fractal growth proceeds and finally vanishes at a surprisingly early stage. This means that the fractal growth "freezes out" on its way to the subsequent growth stage where collisional compression of aggregates occurs. This is because the electrostatic repulsion between aggregates becomes strong enough to prevent their mutual collision. Strong turbulence in the disk will help the aggregates to overcome this electric barrier, but then it will cause catastrophic disruption of collided aggregates at later stages. Therefore, if the freeze-out of the fractal growth truly means the end of dust evolution, the combination of the electric charging and the collisional disruption imposes a very strict limitation on dust coagulation and subsequent planetesimal formation in protoplanetary disks. Our findings strongly suggest that the dust charing effect should be seriously taking into account in the modeling of dust evolution.

This paper is organized as follows. In $\S 2$, we present a set of equations that describes the reactions of charged particles (ions, electrons, and dust aggregates), and derive the equation that determines the equilibrium state. In $\$ 3$, we calculate the electrostatic repulsion energy between two colliding aggregates to show that the quasi-monodisperse fractal growth is strongly inhibited for a wide range of disk parameters. In $\S 4$, we discuss the validity of some important assumptions and point out a possible scenario of dust evolution after the "freeze-out" of the fractal growth. A summary is presented in $\S 5$.

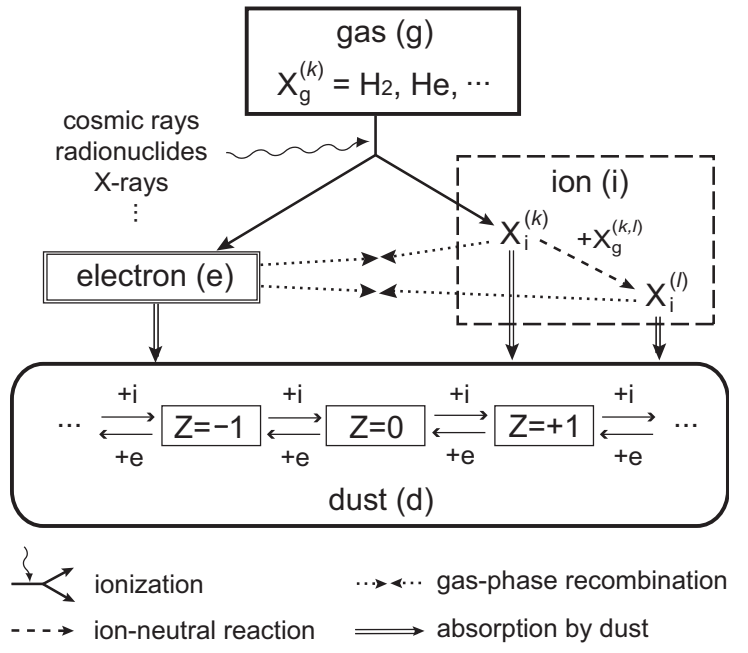

FIG. 1.- Schematic diagram of the ionization-recombination reactions in a gas-dust mixture. Ions and free electrons are created (solid arrows) by some ionizing sources (e.g., cosmic rays) and are removed through the gas-phase recombination (dotted arrows) or the adsorption to dust (double line arrows). Some species of ions may react with neutral gas particles to create different species of ions (dashed arrows). The equilibrium charge distribution $n_{\mathrm{d}}(Z)$ of dust aggregates are determined by the balance between all these reactions.

\section{EQUILIBRIUM CHARGE DISTRIBUTION}

\subsection{Kinetic equations for ionization-recombination reactions}

We model the ionization-recombination reactions in a gasdust mixture as follows (see also fig 1). Some ionizing sources (e.g. cosmic rays) create ions $\mathrm{X}_{\mathrm{i}}^{(k)}$ and free electrons from neutral particles $\mathrm{X}_{\mathrm{g}}^{(k)}$ at a rate $\zeta^{(k)}$ (here $k(=1,2, \cdots)$ labels each species of ions and associated neutrals). We assume that the ions and electrons are quickly thermalized and have thermal velocities $u_{\mathrm{i}}^{(k)}$ and $u_{\mathrm{e}}$, respectively. We neglect the possibility that the free electrons might be much more energetic in a MRI-active region (Inutsuka \& Sano 2005). The ions may react with neutrals $\mathrm{X}_{\mathrm{g}}^{(k, l)}$ to produce another species of ions $\mathrm{X}_{\mathrm{i}}^{(l)}(l \neq k)$, or may recombine with free electrons in the gas phase. We denote the rate coefficient for the ion-neutral reaction and the gas-phase recombination by $\beta^{(k, l)}$ and $\beta^{(k)}$, respectively. Also, the ions and free electrons may collide with dust aggregates to adsorb onto their surfaces. We write the collisional cross section for an aggregate and an ion (electron) as $\sigma_{\mathrm{di}(\mathrm{de})}$. These cross sections generally include the effect of electrostatic interaction as well as the sticking probability (see \$2.2). Each dust aggregate may have different internal structure and charge $\mathrm{Ze}$ from the other. We represent a set of parameters describing the structure (e.g., mass, radius) as $I=\left\{I_{1}, I_{2}, \cdots\right\}$. In this section, we assume that the above reactions proceeds faster than the mutual collision of dust aggregates and treat $I$ as constant parameters. The validity of this assumption is discussed in $\$ 4.1$.

The above charge reactions are described by a set of kinetic equations. Let us denote the number densities of ions $\mathrm{X}_{\mathrm{i}}^{(k)}$, free electrons, and dust aggregates as $n_{\mathrm{i}}^{(k)}, n_{\mathrm{e}}$, and $n_{\mathrm{d}}(I, Z)$, respectively. The rate equations for $n_{\mathrm{i}}^{(k)}, n_{\mathrm{e}}$, and $n_{\mathrm{d}}(I, Z)$ are given by

$$
\dot{n}_{\mathrm{i}}^{(k)}=\zeta^{(k)} n_{\mathrm{g}}^{(k)}-u_{\mathrm{i}}^{(k)} n_{\mathrm{i}}^{(k)} \int d I \sum_{Z} \sigma_{\mathrm{di}}(I, Z) n_{\mathrm{d}}(I, Z)
$$




$$
\begin{aligned}
& -\beta^{(k)} n_{\mathrm{i}}^{(k)} n_{\mathrm{e}}-\sum_{l}\left[\beta^{(k, l)} n_{\mathrm{i}}^{(k)} n_{\mathrm{g}}^{(k, l)}-\beta^{(l, k)} n_{\mathrm{i}}^{(l)} n_{\mathrm{g}}^{(l, k)}\right], \\
\dot{n}_{\mathrm{e}} & =\sum_{k} \zeta^{(k)} n_{\mathrm{g}}^{(k)}-u_{\mathrm{e}} n_{\mathrm{e}} \int d I \sum_{Z} \sigma_{\mathrm{de}}(I, Z) n_{\mathrm{d}}(I, Z) \\
& -\sum_{k} \beta^{(k)} n_{\mathrm{i}}^{(k)} n_{\mathrm{e}}
\end{aligned}
$$

and

$$
\begin{aligned}
\dot{n}_{\mathrm{d}}(I, Z)= & \sum_{k} u_{\mathrm{i}}^{(k)} n_{\mathrm{i}}^{(k)}\left[\sigma_{\mathrm{di}}(I, Z-1) n_{\mathrm{d}}(I, Z-1)-\sigma_{\mathrm{di}}(I, Z) n_{\mathrm{d}}(I, Z)\right] \\
& +u_{\mathrm{e}} n_{\mathrm{e}}\left[\sigma_{\mathrm{de}}(I, Z+1) n_{\mathrm{d}}(I, Z+1)-\sigma_{\mathrm{de}}(I, Z) n_{\mathrm{d}}(I, Z)\right],(3)
\end{aligned}
$$

respectively. In equations (1) and (2), $n_{\mathrm{g}}^{(k)}$ and $n_{\mathrm{g}}^{(k, l)}$ denote the number densities of neutrals $X_{\mathrm{g}}^{(k)}$ and $\mathrm{X}_{\mathrm{g}}^{(k, l)}$. We assume that neutral particles are much more abundant than charged particles and regard $n_{\mathrm{g}}^{(k)}$ and $n_{\mathrm{g}}^{(k, l)}$ as constant parameters.

In addition, we impose the charge neutrality condition

$$
\sum_{k} n_{\mathrm{i}}^{(k)}-n_{\mathrm{e}}+\int d I \sum_{Z} Z n_{\mathrm{d}}(I, Z)=0 .
$$

Equations (1)-(4) form the closed set of basic equations for ionization-recombination reactions in gas-dust mixture.

\subsection{The equilibrium solution}

The equilibrium solution is obtained by imposing the conditions

$$
\dot{n}_{\mathrm{i}}^{(k)}=\dot{n}_{\mathrm{e}}=\dot{n}_{\mathrm{d}}(I, Z)=0
$$

for all $k, I$, and $Z$.

Usually, the equilibrium solutions are calculated with a reaction scheme involving many ion species and ion-neutral reactions (e.g., Umebayashi \& Nakano 1980; Ilgner \& Nelson 2006a). Because of the complexity of the ion-neutral reactions, it is generally impossible to solve this problem analytically without any approximation or simplification.

This problem, however, becomes analytically tractable if we dot not require to distinguish ion species. This is just achieved by introducing the total ion density

$$
n_{\mathrm{i}}=\sum_{k} n_{\mathrm{i}}^{(k)}
$$

Taking the sum of equations (1) over all $k$, we obtain the rate equation for $n_{\mathrm{i}}$,

$$
\dot{n}_{\mathrm{i}}=\zeta n_{\mathrm{g}}-u_{\mathrm{i}} n_{\mathrm{i}} \int d I \sum_{Z} \sigma_{\mathrm{di}}(I, Z) n_{\mathrm{d}}(I, Z)-\beta n_{\mathrm{i}} n_{\mathrm{e}}
$$

where $n_{\mathrm{g}}=\sum_{k} n_{\mathrm{g}}^{(k)}$ is the total number density of the gas, and

$$
\begin{aligned}
& u_{\mathrm{i}}=\frac{1}{n_{\mathrm{i}}} \sum_{k} n_{\mathrm{i}}^{(k)} u_{\mathrm{i}}^{(k)}, \\
& \beta=\frac{1}{n_{\mathrm{i}}} \sum_{k} n_{\mathrm{i}}^{(k)} \beta^{(k)}, \\
& \zeta=\frac{1}{n_{\mathrm{g}}} \sum_{k} n_{\mathrm{g}}^{(k)} \zeta^{(k)},
\end{aligned}
$$

are the average ion velocity, gas-phase recombination rate coefficient, and ionization rate, respectively. Similarly, equations (2) - (4) can be also written down in terms of $n_{\mathrm{i}}, u_{\mathrm{i}}, \beta$, and $\zeta$. It is evident that equation (7) is much simpler than the original equation (1). This is mainly attributed to the cancellation of the last term in the original equation, i.e., the term describing the ion-neutral reactions. Of course, this also means that we have lost the chance to know the composition of ions in detail. This fact does not bother us since our primary interest is the charge state of dust aggregates, not the composition of ions.

Now we try to solve the equations (2)-(4) and (7) under the equilibrium condition (5) as analytically as possible. First, equations (7) and (2) are written as

$$
\begin{gathered}
\zeta n_{\mathrm{g}}-u_{\mathrm{i}} \overline{\left\langle\sigma_{\mathrm{di}}\right\rangle} n_{\mathrm{d}} n_{\mathrm{i}}-\beta n_{\mathrm{i}} n_{\mathrm{e}}=0, \\
\zeta n_{\mathrm{g}}-u_{\mathrm{e}} \overline{\left\langle\sigma_{\mathrm{de}}\right\rangle} n_{\mathrm{d}} n_{\mathrm{e}}-\beta n_{\mathrm{i}} n_{\mathrm{e}}=0,
\end{gathered}
$$

respectively. Here we have defined the averages of an arbitrary function $F=F(I, Z)$ over $Z$ and over $I$ as

$$
\begin{gathered}
\langle F\rangle(I) \equiv \frac{1}{n_{\mathrm{d}}(I)} \sum_{Z} F(I, Z) n_{\mathrm{d}}(I, Z) \\
\bar{F}(Z) \equiv \frac{1}{n_{\mathrm{d}}(Z)} \int F(I, Z) n_{\mathrm{d}}(I, Z) d I
\end{gathered}
$$

with $n_{\mathrm{d}}(I) \equiv \sum_{Z} n_{\mathrm{d}}(I, Z)$ and $n_{\mathrm{d}}(Z) \equiv \int n_{\mathrm{d}}(I, Z) d I$, respectively. We have also defined the total number density $n_{\mathrm{d}}$ of dust aggregates by $n_{\mathrm{d}}=\sum_{Z} \int n_{\mathrm{d}}(I, Z) d I$. Equations (11) and (12) can be easily solved in terms of $n_{\mathrm{i}}$ and $n_{\mathrm{e}}$ as

$$
\begin{aligned}
& n_{\mathrm{i}}=\frac{u_{\mathrm{e}} \overline{\left\langle\sigma_{\mathrm{de}}\right\rangle} n_{\mathrm{d}}}{2 \beta}\left(\sqrt{1+\frac{4 \beta \zeta n_{\mathrm{g}}}{u_{\mathrm{i}} u_{\mathrm{e}} \overline{\left\langle\sigma_{\mathrm{di}}\right\rangle} \overline{\left\langle\sigma_{\mathrm{de}}\right\rangle} n_{\mathrm{d}}^{2}}}-1\right), \\
& n_{\mathrm{e}}=\frac{u_{\mathrm{i}} \overline{\left\langle\sigma_{\mathrm{di}}\right\rangle} n_{\mathrm{d}}}{2 \beta}\left(\sqrt{1+\frac{4 \beta \zeta n_{\mathrm{g}}}{u_{\mathrm{i}} u_{\mathrm{e}} \overline{\left\langle\sigma_{\mathrm{di}}\right\rangle} \overline{\left\langle\sigma_{\mathrm{de}}\right\rangle} n_{\mathrm{d}}^{2}}}-1\right) .
\end{aligned}
$$

Next, equation (3) is reduced to

$$
\begin{aligned}
& u_{\mathrm{i}} n_{\mathrm{i}} \sigma_{\mathrm{di}}(I, Z-1) n_{\mathrm{d}}(I, Z-1)-u_{\mathrm{e}} n_{\mathrm{e}} \sigma_{\mathrm{de}}(I, Z) n_{\mathrm{d}}(I, Z) \\
& =u_{\mathrm{i}} n_{\mathrm{i}} \sigma_{\mathrm{di}}(I, Z) n_{\mathrm{d}}(I, Z)-u_{\mathrm{e}} n_{\mathrm{e}} \sigma_{\mathrm{de}}(I, Z+1) n_{\mathrm{d}}(I, Z+1) .
\end{aligned}
$$

This equation means that the "flux" of the distribution $n_{\mathrm{d}}(I, Z)$ from the state $Z-1$ to $Z$ must be balanced to that from $Z$ to $Z+1$. If the flux did not vanish, there would exist a steady "flow" of the charge state distribution $n_{\mathrm{d}}(I, Z)$ streaming from one direction to the other in $Z$-space. However, since $n_{\mathrm{d}}(I, Z)$ must be vanish at $Z \rightarrow \pm \infty$, such a steady flow must not exist. Therefore, both of the left and right-hand sides of equation (17) must be zero. Hence we have

$$
u_{\mathrm{i}} n_{\mathrm{i}} \sigma_{\mathrm{di}}(I, Z) n_{\mathrm{d}}(I, Z)=u_{\mathrm{e}} n_{\mathrm{e}} \sigma_{\mathrm{de}}(I, Z+1) n_{\mathrm{d}}(I, Z+1) .
$$

This is just the condition of detailed balance between charge states $Z$ and $Z+1$.

For the sake of later convenience, we here rewrite the charge neutrality condition (4) using the definitions (6), (13), and (14) as

$$
n_{\mathrm{i}}-n_{\mathrm{e}}+\overline{\langle Z\rangle} n_{\mathrm{d}}=0
$$

As we will see later, this is the final equation that determines the equilibrium solution.

The next step is to solve the detailed balance equation (18). To carry out the calculation, we need to specify the forms of the effective collision cross sections, $\sigma_{\mathrm{di}}$ and $\sigma_{\mathrm{de}}$. For simplicity, we model a fractal aggregate as a spherical, porous body with radius $a$ and projected cross section $\sigma$. Also, we neglect the electric polarization of aggregates (Draine \& Sutin 
1987) since a fractal aggregate is likely to have low dielectricity. Draine \& Sutin (1987) found that the strength of the electric polarization relative to the charge-charge (Coulomb) interaction is determined by a factor $(\epsilon-1) /(\epsilon+2)$, where $\epsilon$ is the dielectric constant of the material. Given that a porous aggregate is well approximated by a dilute medium, the factor $(\epsilon-1) /(\epsilon+2)$ is inversely proportional to its mean internal density (Clausius-Mossotti relation; see, e.g., Jackson 1998). For example, the mean internal density of a fractal aggregate with $D \approx 2$ is about inversely proportional to its radius $a$. Therefore, we expect that the polarization effect of such a low-density aggregate is negligible. Neglecting the polarization effect, the effective cross sections are simply written as (Spitzer 1941)

$$
\begin{gathered}
\sigma_{\mathrm{di}}(I, Z)=\sigma s_{\mathrm{i}}(Z) \times \begin{cases}\left(1-\frac{\lambda Z}{a}\right), & Z<0, \\
\exp \left(-\frac{\lambda Z}{a}\right), & Z \geq 0,\end{cases} \\
\sigma_{\mathrm{de}}(I, Z)=\sigma s_{\mathrm{e}}(Z) \times \begin{cases}\exp \left(\frac{\lambda Z}{a}\right), & Z<0, \\
\left(1+\frac{\lambda Z}{a}\right), & Z \geq 0,\end{cases}
\end{gathered}
$$

where $s_{\mathrm{i}(\mathrm{e})}(Z)$ is the probability that a colliding ion (electron) sticks to one of constituent monomers, and $\lambda=e^{2} / k_{\mathrm{B}} T$. In this study, we assume that $s_{\mathrm{i}}(Z)$ and $s_{\mathrm{e}}(Z)$ are independent of the net charge $Z$ carried by an aggregate, i.e., $s_{\mathrm{i}(\mathrm{e})}(Z) \equiv s_{\mathrm{i}(\mathrm{e})}$.

As shown in Appendix, the solution $n_{\mathrm{d}}(I, Z)$ to equation (18) with equations (20) and (21) is well approximated by a Gaussian distribution

$$
n_{\mathrm{d}}(I, Z)=\frac{n_{\mathrm{d}}(I)}{\sqrt{2 \pi\left\langle\Delta Z^{2}\right\rangle_{a}}} \exp \left[-\frac{\left(Z-\langle Z\rangle_{a}\right)^{2}}{2\left\langle\Delta Z^{2}\right\rangle_{a}}\right],
$$

where

$$
\langle Z\rangle_{a} \equiv \frac{1}{n_{\mathrm{d}}(I)} \sum_{Z} Z n_{\mathrm{d}}(I, Z) \equiv-\frac{\Gamma a}{\lambda}
$$

and

$$
\left\langle\Delta Z^{2}\right\rangle_{a} \equiv \frac{1}{n_{\mathrm{d}}(I)} \sum_{Z}\left(Z-\langle Z\rangle_{a}\right)^{2} n_{\mathrm{d}}(I, Z)=\frac{1+\Gamma}{2+\Gamma} \frac{a}{\lambda}
$$

are the mean and dispersion of the charge distribution for fixed $a$, respectively. This solution is valid when the radius $a$ is much larger than $\lambda$, as is for aggregates much larger than constituent monomers (see Appendix). The nondimensional parameter $\Gamma \equiv-\langle Z\rangle \lambda / a=\left(-\langle Z\rangle e^{2} / a\right) / k_{\mathrm{B}} T$ measures the electrostatic attraction (repulsion) energy between a charged aggregate and an incident ion (electron) relative to the thermal kinetic energy.

Since the charge distribution is parametrized by $\Gamma$ only, $n_{\mathrm{i}}$ and $n_{\mathrm{e}}$ can be written as a function of a single parameter $\Gamma$. Using equations (22)-24), $\left\langle\sigma_{\mathrm{di}}\right\rangle$ and $\left\langle\sigma_{\mathrm{de}}\right\rangle$ are evaluated as

$$
\begin{gathered}
\left\langle\sigma_{\mathrm{di}}\right\rangle=\sigma s_{\mathrm{i}}(1+\Gamma), \\
\left\langle\sigma_{\mathrm{de}}\right\rangle=\sigma s_{\mathrm{e}} \exp \left[-\Gamma+\frac{\lambda(1+\Gamma)}{2 a(2+\Gamma)}\right] \approx \sigma s_{\mathrm{e}} \exp (-\Gamma),
\end{gathered}
$$

respectively. Here we have used in equation (26) that $a / \lambda \gg$ 1. Substituting equations (25) and (26) into equations (15) and (16), we have

$$
n_{\mathrm{i}}=\frac{\zeta n_{\mathrm{g}}}{s_{\mathrm{i}} u_{\mathrm{i}} \bar{\sigma} n_{\mathrm{d}}} \frac{\sqrt{1+2 g(\Gamma)}-1}{(1+\Gamma) g(\Gamma)}
$$

$$
n_{\mathrm{e}}=\frac{\zeta n_{\mathrm{g}}}{s_{\mathrm{e}} u_{\mathrm{e}} \bar{\sigma} n_{\mathrm{d}}} \frac{\sqrt{1+2 g(\Gamma)}-1}{\exp (-\Gamma) g(\Gamma)}
$$

where

$$
g(\Gamma)=\frac{2 \beta \zeta n_{\mathrm{g}}}{s_{\mathrm{i}} u_{\mathrm{i}} s_{\mathrm{e}} u_{\mathrm{e}}\left(\bar{\sigma} n_{\mathrm{d}}\right)^{2}} \frac{\exp \Gamma}{1+\Gamma} .
$$

Finally, the neutrality condition (19) with equations (23), (27), and 28) leads to the equation for $\Gamma$,

$$
\frac{1}{1+\Gamma}-\left[\frac{s_{\mathrm{i}} u_{\mathrm{i}}}{s_{\mathrm{e}} u_{\mathrm{e}}} \exp \Gamma+\frac{1}{\Theta} \frac{\Gamma g(\Gamma)}{\sqrt{1+2 g(\Gamma)}-1}\right]=0,
$$

where we have defined a nondimensional parameter

$$
\Theta \equiv \frac{\zeta n_{\mathrm{g}} \lambda}{s_{\mathrm{i}} u_{\mathrm{i}} \bar{\sigma} \bar{a} n_{\mathrm{d}}^{2}}=\frac{\zeta n_{\mathrm{g}} e^{2}}{s_{\mathrm{i}} u_{\mathrm{i}} \bar{\sigma} \bar{a} n_{\mathrm{d}}^{2} k_{\mathrm{B}} T} .
$$

We have consequently arrived at the conclusion that all the conditions for ionization equilibrium, equations (11), (12), and (18), are reduced to a single equation (30) for a single parameter $\Gamma$.

To summarize the above analysis, we have considered the charge state of a dust-gas mixture in the presence of ionization sources. We have found that the self-consistent equilibrium solutions are written as analytical functions of a single parameter $\Gamma$ (eqs. [22]-[24], 27], and [28]), and have obtained the equation for this master parameter (eq. [30]). This equation can be easily solved numerically, and thus gives semianalytical solutions to $n_{\mathrm{i}}, n_{\mathrm{e}}$, and $n_{\mathrm{d}}(I, Z)$.

The resultant equations can be further simplified when the gas-phase recombination rate coefficient $\beta$ is so small that the factor $g$ defined in equation (29) is much less than unity. In this limit, equations (27), (28), and (30) are simply rewritten as

$$
\begin{aligned}
& n_{\mathrm{i}}=\frac{\zeta n_{\mathrm{g}}}{s_{\mathrm{i}} u_{\mathrm{i}} \bar{\sigma} n_{\mathrm{d}}} \frac{1}{1+\Gamma}, \\
& n_{\mathrm{e}}=\frac{\zeta n_{\mathrm{g}}}{s_{\mathrm{e}} u_{\mathrm{e}} \bar{\sigma} n_{\mathrm{d}}} \exp \Gamma,
\end{aligned}
$$

and

$$
\frac{1}{1+\Gamma}-\left[\frac{s_{\mathrm{i}} u_{\mathrm{i}}}{s_{\mathrm{e}} u_{\mathrm{e}}} \exp \Gamma+\frac{\Gamma}{\Theta}\right]=0,
$$

respectively. As seen in $\$ 3.1 .3$, this approximation is valid in typical protoplanetary disks unless the dust is significantly depleted (e.g., by vertical sedimentation).

\subsection{Limiting cases}

Equation (30), or (34), provides clear insight into the charge state of a gas-dust mixture. The first and second terms in the bracket in this equation originate from $n_{\mathrm{e}}$ and $-\langle Z\rangle n_{\mathrm{d}}$ in the charge neutrality condition (4), respectively. This means that the parameter $\Theta$ determines which of free electrons and dust aggregates are the dominant carriers of negative charge. In the following, we categorize the charge state from limiting cases of equation (34).

\subsection{1. $\Theta \rightarrow \infty$ : ion-electron plasma limit}

In the limit $\Theta \rightarrow \infty$, the contribution from charged dust becomes negligibly small. Hence, equation (34) is well approximated by

$$
\frac{1}{1+\Gamma} \approx \frac{s_{\mathrm{i}} u_{\mathrm{i}}}{s_{\mathrm{e}} u_{\mathrm{e}}} \exp \Gamma .
$$

This is a well-known charge-equilibrium condition for a dust particle immersed in an ordinary ion-electron plasma (Spitzer 
1941; Shukla \& Mamun 2002). We denote the solution $\Gamma$ to equation (35) by $\Gamma_{\max }$, since this is the maximum value of $\Gamma$ obtained from equation (34). Typically, $\Gamma_{\max }$ takes a value of order unity.

\subsection{2. $\Theta \rightarrow 0$ : ion-dust plasma limit}

In the limit $\Theta \rightarrow 0$, on the other hand, the contribution from free electrons becomes negligible. This means that the dominant carriers of negative charges are dust particles, not free electrons. We shall refer to this limit as the ion-dust plasma limit. In this limit, equation (34) can be approximated by

$$
\frac{1}{1+\Gamma} \approx \frac{\Gamma}{\Theta} \text {. }
$$

The solution is easily obtained as

$$
\Gamma \approx \frac{\sqrt{1+4 \Theta^{2}}-1}{2} \approx \Theta .
$$

Since $\Gamma$ is now negligibly small, the effective cross sections $\left\langle\sigma_{\mathrm{di}(\mathrm{e})}\right\rangle$ are approximately equal to $\sigma s_{\mathrm{i}(\mathrm{e})}$. Equations (32) and (33) lead to the ratio of $n_{\mathrm{e}}$ to $n_{\mathrm{i}}$,

$$
\frac{n_{\mathrm{e}}}{n_{\mathrm{i}}} \approx \frac{s_{\mathrm{i}} u_{\mathrm{i}}}{s_{\mathrm{e}} u_{\mathrm{e}}} \approx \frac{s_{\mathrm{i}}}{s_{\mathrm{e}}} \sqrt{\frac{m_{\mathrm{e}}}{m_{\mathrm{i}}}} .
$$

where we have used that $u_{\mathrm{i}(\mathrm{e})}=\sqrt{8 k_{\mathrm{B}} T / \pi m_{\mathrm{i}(\mathrm{e})}}$. We note that this value of $n_{\mathrm{e}} / n_{\mathrm{i}}$ is larger than that of Umebayashi (1983) by a factor of $1 / \sqrt{s_{\mathrm{e}}}$. This is because we have assumed the value of $s_{\mathrm{e}}$ as independent of $Z$ while Umebayashi (1983) considered $s_{\mathrm{e}}(Z>0)=1$. As far as the author knows, there is no experimental data that validates either of the assumptions. However, this difference is practically unimportant unless the ratio $\sqrt{s_{\mathrm{e}}(Z<0) / s_{\mathrm{e}}(Z \geq 0)}$ is much less than unity.

The value of $\Theta$ at the transition from one plasma regime to the other can be roughly estimated by equating the asymptotic solutions for both limits, i.e., $\Theta \approx \Gamma_{\max }$. We will use this estimation in the next section.

\section{APPLICATION: ELECTRIC BARRIER AGAINST DUST GROWTH}

Electrostatic interaction between charged aggregates affect their collisional cross section. Let us consider two dust aggregates with mass $m_{j}$, radius $a_{j}$ and charge $Z_{j} e$, where $j(=1,2)$ labels the aggregates. The kinetic energy for relative motion of two aggregates 1 and 2 is written as

$$
E_{\mathrm{kin}}=\frac{1}{2} \tilde{m}(\Delta u)^{2},
$$

where $\tilde{m}=m_{1} m_{2} /\left(m_{1}+m_{2}\right)$ and $\Delta u$ are the reduced mass and the relative speed for the aggregate pair. The electrostatic energy between the aggregates just before contact is

$$
E_{\mathrm{el}}=\frac{Z_{1} Z_{2} e^{2}}{a_{1}+a_{2}} .
$$

Neglecting the polarization effect as done in $\S 2$, the effective collision cross section $\sigma_{\mathrm{dd}}$ for the aggregates is expressed as Spitzer 1941)

$$
\sigma_{\mathrm{dd}}= \begin{cases}\pi\left(a_{1}+a_{2}\right)^{2}\left(1-\frac{E_{\mathrm{el}}}{E_{\mathrm{kin}}}\right), & E_{\mathrm{kin}}>E_{\mathrm{el}}, \\ 0, & E_{\mathrm{kin}} \leq E_{\mathrm{el}},\end{cases}
$$

Therefore, the condition for the aggregates to collide with each other is

$$
E_{\text {kin }}>E_{\text {el }} \text {. }
$$

In this section, we examine whether this condition is satisfied in an early stage of dust evolution in a protoplanetary disk.

\subsection{Model setup}

\subsubsection{Protoplanetary disk model}

We assume that the gas surface density $\Sigma_{\mathrm{g}}$ and the temperature $T$ of the disk obey power laws

$$
\Sigma_{\mathrm{g}}(r)=1.7 \times 10^{3} f_{\Sigma}\left(\frac{r}{1 \mathrm{AU}}\right)^{-3 / 2} \mathrm{~g} / \mathrm{cm}^{2},
$$

and

$$
T(r)=280\left(\frac{r}{1 \mathrm{AU}}\right)^{-1 / 2} \mathrm{~K},
$$

where $r$ is the distance from the central star and $f_{\Sigma}$ is a nondimensional scaling parameter. The model with $f_{\Sigma}=1$ is known as the minimum-mass solar nebula (MMSN) model (Hayashi 1981). We adopt $f_{\Sigma}=1$ unless otherwise noted. The temperature profile (44) is valid only for optically thin regions. We nevertheless employ this profile throughout the disk since the main result is insensitive to the detail of the temperature profile (see eq. [65] below).

The hydrostatic equilibrium in the vertical direction gives the gas density distribution

$$
\rho_{\mathrm{g}}(r, z)=\frac{\Sigma_{\mathrm{g}}}{\sqrt{2 \pi} H(r)} \exp \left[-\frac{z^{2}}{2 H(r)^{2}}\right],
$$

where $z$ is the height from the disk midplane and $H(r)=$ $c_{s}(r) / \Omega_{\mathrm{K}}(r)$ is the gas scale height. The isothermal sound velocity $c_{s}$ and the Kepler rotational frequency $\Omega_{\mathrm{K}}(r)$ are given by $c_{s}(r)=\sqrt{k_{\mathrm{B}} T(r) / \mu m_{\mathrm{H}}}$ and $\Omega_{\mathrm{K}}(r)=\sqrt{G M_{*} / r^{3}}$, where $\mu$ is the mean molecular weight, $m_{\mathrm{H}}$ is the hydrogen mass, $G$ is the gravitational constant, and $M_{*}$ is the mass of the central star. We adopt $\mu=2.34$ and $M_{*}=1 M_{\odot}$ in the following calculation. The total number density $n_{\mathrm{g}}$ of gas particles is given by $n_{\mathrm{g}}=\rho_{\mathrm{g}} / \mu m_{\mathrm{H}}$.

We assume that dust material is well mixed in the disk and that the dust density $\rho_{\mathrm{d}}$ is simply related to the gas density $\rho_{\mathrm{g}}$ by

$$
\rho_{\mathrm{d}}(r, z)=f_{\mathrm{dg}} \rho_{\mathrm{g}}(r, z),
$$

where $f_{\mathrm{dg}}$ is the dust-to-gas ratio in the disk. The solar system abundance of condensates including water ice estimated by Pollack et al. (1994) leads to the dust-to-gas ratio $f_{\mathrm{dg}}=$ 0.014 as well as the monomer bulk density $\rho_{0}=1.4 \mathrm{~g} / \mathrm{cm}^{3}$ (Tanaka et al. 2005). We do not consider the sublimation of water ice in inner disk regions for simplicity.

\subsubsection{Ionization rate}

In this study, we consider Galactic cosmic rays (Umebavashi \& Nakano 1981), stellar Xrays (Igea \& Glassgold 1999), and radionuclides (Umebayashi \& Nakano 2009) as the ionizing sources. Thus, we decompose the ionization rate $\zeta$ as

$$
\zeta \approx \zeta_{\mathrm{CR}}+\zeta_{\mathrm{XR}}+\zeta_{\mathrm{RA}}
$$

where $\zeta_{\mathrm{CR}}, \zeta_{\mathrm{XR}}$, and $\zeta_{\mathrm{RA}}$ denote the rate of ionization by cosmic rays, X-rays, and radionuclides, respectively. We do not consider thermal ionization. This is negligible for $T \ll 10^{3} \mathrm{~K}$, or for $r \gg 0.1 \mathrm{AU}$ (Umebavashi 1983). Charged particles are created primarily by ionization of $\mathrm{H}_{2}$ and He. The ionization rate for He is related to that for $\mathrm{H}_{2}$ by $\zeta^{(\mathrm{He})}=0.84 \zeta^{\left(\mathrm{H}_{2}\right)}$ (Umebayashi \& Nakano 1990, 2009), so it is sufficient to know $\zeta^{\left(\mathrm{H}_{2}\right)}$ only. The total ionization rate $\zeta$ is given by $\zeta=$ $\zeta^{\left(\mathrm{H}_{2}\right)} x_{\mathrm{H}_{2}}+\zeta^{(\mathrm{He})} x_{\mathrm{He}}$, where $x_{\mathrm{H}_{2}}=n_{\mathrm{H}_{2}} / n_{\mathrm{g}}$ and $x_{\mathrm{He}}=n_{\mathrm{He}} / n_{\mathrm{g}}$ are the fractional abundances of $\mathrm{H}_{2}$ and $\mathrm{He}$. We calculate $x_{\mathrm{H}_{2}}$ and 
$x_{\mathrm{He}}$ from the solar system abundance by Anders \& Grevesse (1989).

The cosmic-ray ionization rate $\zeta_{\mathrm{CR}}^{\left(\mathrm{H}_{2}\right)}$ for $\mathrm{H}_{2}$ is given by a fitting formula (Umebayashi \& Nakano 2009)

$$
\begin{aligned}
\zeta_{\mathrm{CR}}^{\left(\mathrm{H}_{2}\right)}(r, z) \approx & \frac{\zeta_{\mathrm{CR}, 0}^{\left(\mathrm{H}_{2}\right)}}{2}\left\{\exp \left(-\frac{\chi_{\mathrm{g}}^{+}(r, z)}{\chi_{\mathrm{CR}}}\right)\left[1+\left(\frac{\chi_{\mathrm{g}}^{+}(r, z)}{\chi_{\mathrm{CR}}}\right)^{3 / 4}\right]^{-4 / 3}\right. \\
& \left.+\exp \left(-\frac{\chi_{\mathrm{g}}^{-}(r, z)}{\chi_{\mathrm{CR}}}\right)\left[1+\left(\frac{\chi_{\mathrm{g}}^{-}(r, z)}{\chi_{\mathrm{CR}}}\right)^{3 / 4}\right]^{-4 / 3}\right\},(48)
\end{aligned}
$$

where $\zeta_{\mathrm{CR}, 0}^{\left(\mathrm{H}_{2}\right)} \approx 1.0 \times 10^{-17} / \mathrm{s}$ is the cosmic-ray ionization rate for $\mathrm{H}_{2}$ in the interstellar space, $\chi_{\mathrm{CR}} \approx 96 \mathrm{~g} / \mathrm{cm}^{2}$ is the attenuation length of the ionization rate, and $\chi_{\mathrm{g}}^{+}(r, z)=\int_{z}^{\infty} \rho_{\mathrm{g}}\left(r, z^{\prime}\right) d z^{\prime}$ and $\chi_{\mathrm{g}}^{-}(r, z)=\Sigma_{\mathrm{g}}(r)-\chi_{\mathrm{g}}^{+}(r, z)$ are the vertical gas column densities measured from the upper and lower infinities, respectively. For the radionuclide ionization rate, we assume $\zeta_{\mathrm{RA}}^{\left(\mathrm{H}_{2}\right)} \approx 7 \times 10^{-19} / \mathrm{s}$, which corresponds to the ionization rate by ${ }^{26} \mathrm{Al}$ with an abundance ratio ${ }^{26} \mathrm{Al} /{ }^{27} \mathrm{Al}=5 \times 10^{-5}$ (Umebayashi \& Nakano 2009).

The stellar X-ray ionization rate has been calculated by Igea \& Glassgold (1999) using the Monte Carlo radiative transfer code including Compton scattering. A useful fitting formula is given by Turner \& Sano (2008),

$$
\begin{aligned}
\zeta_{\mathrm{XR}}(r, z) \approx & \zeta_{\mathrm{XR}, 0}\left(\frac{r}{1 \mathrm{AU}}\right)^{-2}\left(\frac{L_{\mathrm{XR}}}{2 \times 10^{30} \mathrm{erg} / \mathrm{s}}\right) \\
& \times\left\{\exp \left(-\frac{\chi_{\mathrm{g}}^{+}(r, z)}{\chi_{\mathrm{XR}}}\right)+\exp \left(-\frac{\chi_{\mathrm{g}}^{-}(r, z)}{\chi_{\mathrm{XR}}}\right)\right\},
\end{aligned}
$$

where $L_{\mathrm{XR}}$ is the X-ray luminosity, and $\zeta_{\mathrm{XR}, 0}=2.6 \times 10^{-15} / \mathrm{s}$ and $\chi_{\mathrm{XR}}=8.0 \mathrm{~g} / \mathrm{cm}^{2}$ are the fitting parameters. This fitting formula approximately reproduces the $k_{\mathrm{B}} T_{\mathrm{XR}}=5 \mathrm{keV}$ result of Igea \& Glassgold (1999) within the column density range $\chi_{\mathrm{g}} \gtrsim 1 \mathrm{~g} / \mathrm{cm}^{2}$, where scattered hard ( $\gtrsim 5 \mathrm{keV}$ ) X-rays are responsible for the ionization. We use equation (49) in the following calculation since the typical value of $\chi_{\mathrm{g}}$ is within the above range. We take $L_{\mathrm{XR}}=2 \times 10^{30} \mathrm{erg} / \mathrm{s}$ in accordance with the median characteristic X-ray luminosity observed by Chandra for young solar-mass stars in the Orion Nebula Cluster (Wolk et al. 2005). Although the characteristic $\mathrm{X}$-ray temperature $k_{\mathrm{B}} T_{\mathrm{XR}} \approx 2.4 \mathrm{keV}$ observed by Wolk et al. (2005) is lower than the assumed value of $k_{\mathrm{B}} T_{\mathrm{XR}} \approx 5 \mathrm{keV}$, the choice of the temperature does not significantly affect the resulting ionization rate (Igea \& Glassgold 1999). We do not consider temporary increase in the X-ray luminosity due to stellar flaring (Wolk et al. 2005), since our analytical method assumes stationary ionization processes. As pointed out by Ilgner \& Nelson (2006c), the flaring could quantitatively change the ionization state of the disk. We will examine the effect of the time-dependent flaring on dust growth in the future work.

Figure 2 shows the total ionization rate $\zeta$ as well as its three components $\left(\zeta_{\mathrm{CR}}, \zeta_{\mathrm{XR}}\right.$, and $\left.\zeta_{\mathrm{RA}}\right)$ as a function of $r$ and $z$. Xray ionization is dominant at outer radii ( $r \gtrsim 4 \mathrm{AU})$, while radionuclide ionization dominates at inner radii $(r \lesssim 1 \mathrm{AU})$. Cosmic-ray ionization is important in outer $(r \gtrsim 2 \mathrm{AU})$ and low-altitude $(z \lesssim H)$ regions.

\subsubsection{Dust growth model}

Based on the results of recent laboratory and computer simulations mentioned in $\S 1$, we model the dust growth in protoplanetary disks as follows. We start with monodisperse, nonaggregated dust grains (monomers) with radius $a_{0}$. The mass $m_{0}$ and number density $n_{\mathrm{d} 0}$ of monomers are then written as $m_{0}=(4 \pi / 3) \rho_{0} a_{0}^{3}$ and $n_{\mathrm{d} 0}=\rho_{\mathrm{d}} / m_{0}$. The dust is assumed to grow into an ensemble of quasi-monodisperse, fractal aggregates with typical monomer number $N$ and fractal dimension $D \sim 2$. Under this assumption, we may regard $N$ as the label of dust evolutionary stages. Theoretically, this type of growth is the best modeled by the so-called ballistic clustercluster aggregation (BCCA; e.g., Meakin 1991). For this reason, we shall refer to the quasi-monodisperse fractal growth as the "BCCA growth."1 Note that the number density $n_{\mathrm{d}}$ of aggregates is inversely proportional to $N$, since $n_{\mathrm{d} 0}=N n_{\mathrm{d}}$ is conserved. The fractal growth continues until colliding aggregates become energetic enough to compress each other. According to the microscopic model of Dominik \& Tielens (1997), the critical kinetic energy for the onset of collisional compression is given by $E_{\text {kin }} \sim E_{\text {roll }}$, where

$$
\begin{aligned}
E_{\text {roll }} & =6 \pi^{2} \gamma \frac{a_{0}}{2} \xi_{\text {crit }} \\
& \approx 5.9 \times 10^{-10}\left(\frac{\gamma}{100 \mathrm{erg} / \mathrm{cm}^{2}}\right)\left(\frac{\xi_{\text {crit }}}{2 \AA}\right)\left(\frac{a_{0}}{0.1 \mu \mathrm{m}}\right) \mathrm{erg}
\end{aligned}
$$

is the energy needed to roll a monomer on another monomer in contact by $90^{\circ}, \gamma$ is the surface adhesion energy for the two monomers, and $\xi_{\text {crit }}$ is the critical tangential displacement for starting the rolling. For icy monomers, $\gamma$ is estimated as $\gamma \approx 100 \mathrm{~g} / \mathrm{cm}^{2}$ (Israelachvili 1992) but a realistic value of $\xi_{\text {crit }}$ is unknown. For a conservative estimation, we assume the minimum displacement $\xi_{\text {crit }}=2 \AA$ anticipated by the theory (Dominik \& Tielens 1997), which makes our aggregates the most easily compressed. The assumed value of $E_{\text {roll }}$ is not much different from the experimental value for rocky $\left(\mathrm{SiO}_{2}\right)$ monomers, $E_{\text {roll }} \approx 1.3 \times 10^{-9}\left(a_{0} / 0.1 \mu \mathrm{m}\right) \mathrm{erg}$ (Heim et al. 1999), so the duration of the fractal growth stage is insensitive to our choice of dust material. We restrict our calculation to an early growth stage where the relative kinetic energy $E_{\text {kin }}$ does not exceed the critical rolling energy $E_{\text {roll }}$. by

The radius $a$ of a fractal aggregate is approximately given

$$
a \approx a_{0} N^{1 / D} .
$$

A classical, compact aggregate has $D=3$, while a BCCA cluster has $D \approx 1.9$ (Meakin 1991). We adopt $D=2$ in the following calculation. The projected cross section $\sigma$ of an aggregate is simply set to $\sigma \approx \pi a^{2} \approx \sigma_{0} N^{2 / D} \approx \sigma_{0} N$, where $\sigma_{0}=\pi a_{0}^{2}$ is the geometrical cross section of a monomer. This assumption is consistent with the fact that $\sigma \propto N$ for $D \lesssim$ 2 (Meakin \& Donn 1988; Meakin et al. 1989; Minato et al. 2006). Note that the quantity $\sigma n_{\mathrm{d}}$ is independent of $N$, i.e., conserved for the BCCA growth.

Assuming a quasi-monodisperse size distribution, the kinetic energy (39) is written as

$$
E_{\mathrm{kin}} \approx \frac{1}{4} m(\Delta u)^{2}
$$

1 Exactly speaking, the BCCA (i.e., collision between identical aggregates; see Meakin 1991) can only occur when the relative velocity of aggregates is induced by Brownian motion. When the relative velocity is induced by differential sedimentation, any identical aggregates cannot collide with each other, and therefore a BCCA cluster in its original sense cannot be created. On the other hand, a laboratory experiment by Blum et al. (1998) shows that the outcome of sedimentation-driven coagulation is an ensemble of quasi-monodisperse, fractal aggregates with $D \approx 1.7$, as is for Brownianmotion-driven growth (e.g., Kempf et al. 1999). This may be explained by the fact that the dominant growth mode in differential sedimentation is the collision between similar aggregates (Tanaka et al. 2005). For this reason, this study treats the growth by differential sedimentation as the "BCCA growth." 

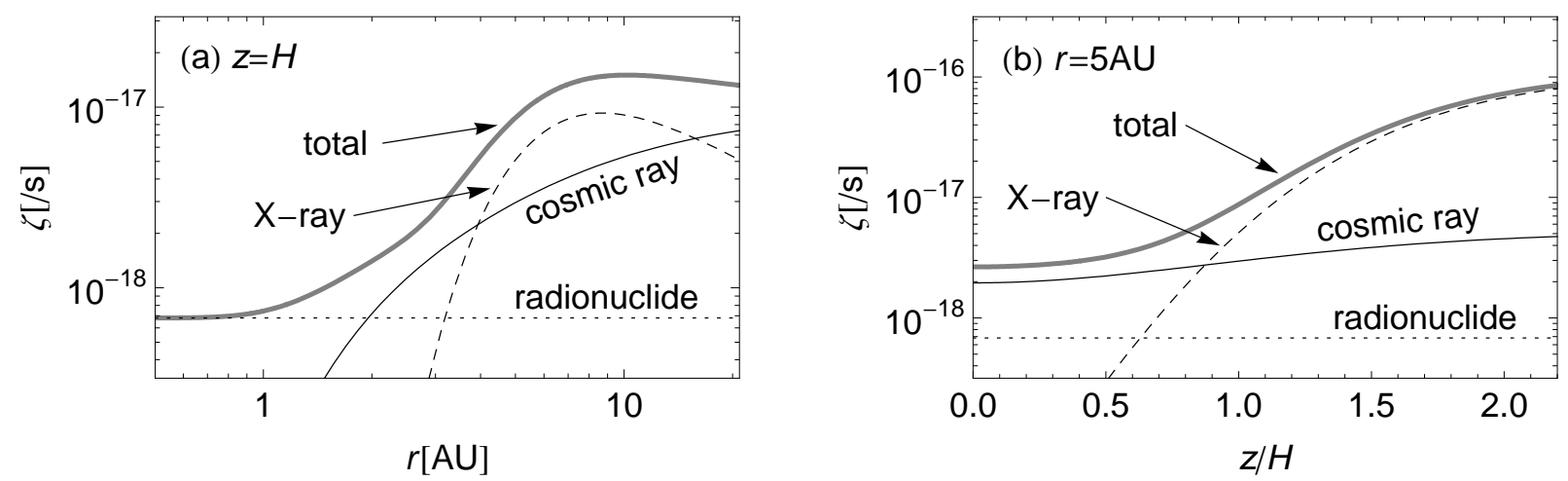

FIG. 2.- Total ionization rate $\zeta$ (thick gray curves) at different disk radii $(a)$ and and altitudes $(b)$. Here $H$ denotes the scale height of the disk. The solid, dashed, and dotted black curves represent the contribution from Galactic cosmic rays, stellar X-rays, and radionuclides, respectively.

where we have used $\tilde{m} \approx m / 2$. In a protoplanetary disk, relative motion of aggregates is induced by Brownian motion, sedimentation toward the midplane of the disk, and turbulence. We therefore write the relative velocity $\Delta u$ as

$$
\Delta u \approx \sqrt{\left(\Delta u_{\text {Brown }}\right)^{2}+\left(\Delta u_{\text {sed }}\right)^{2}+\left(\Delta u_{\text {turb }}\right)^{2}},
$$

where $\Delta u_{\text {Brown }}, \Delta u_{\text {sed }}$, and $\Delta u_{\text {turb }}$ are the relative speed induced by the Brownian motion, differential sedimentation, and turbulence, respectively.

The mean relative speed of the Brownian motion is given by

$$
\Delta u_{\text {Brown }}=\sqrt{\frac{8 k_{\mathrm{B}} T}{\pi \tilde{m}}} \approx \sqrt{\frac{16 k_{\mathrm{B}} T}{\pi m}} .
$$

In fact, the relative speed of the Brownian motion fluctuates according to the Maxwell distribution, and aggregates have a chance to get a relative kinetic energy $E$ much larger than the thermal energy $\sim k_{\mathrm{B}} T$ with a probability $\propto$ $E^{1 / 2} \exp \left(-E / k_{\mathrm{B}} T\right)$. However, as we see later, the effect of the thermal velocity fluctuation is insignificant, since the electrostatic energy can go up to $10^{5} k_{\mathrm{B}} T$.

The relative speed induced by the differential sedimentation is

$$
\Delta u_{\text {sed }}=\Omega_{\mathrm{K}}^{2} z \Delta t_{\text {stop }}
$$

where $t_{\text {stop }}$ is the stopping time of an aggregate. For aggregates smaller than the mean free path $\ell_{\mathrm{g}}$ of gas particles, $t_{\text {stop }}$ is given by Epstein's law

$$
t_{\text {stop }}=\frac{3}{4 \rho_{\mathrm{g}} u_{\mathrm{g}}} \frac{m}{\sigma}
$$

where $u_{\mathrm{g}}=\sqrt{8 k_{\mathrm{B}} T / \pi \mu m_{\mathrm{H}}}$ is the mean thermal speed of gas particles. The mean free path in our disk model is calculated to be $\ell_{\mathrm{g}} \sim 1(r / 1 \mathrm{AU})^{11 / 4} \mathrm{~cm}$, which is much larger than a typical size of aggregate which we are interested in. For this reason, we always use Epstein's law (56) in the following calculation. Also, we replace $\Delta t_{\text {stop }}$ with its maximum value $t_{\text {stop }}$, which leads to the most conservative evaluation of dust charging effect.

The relative velocity induced by turbulence is given by

$$
\Delta u_{\text {turb }} \approx \frac{u_{\text {small }}}{t_{\text {small }}} \Delta t_{\text {stop }}
$$

where $u_{\text {small }}$ and $t_{\text {small }}$ are the characteristic velocity and turnover time of the smallest turbulent eddies, respectively (Weidenschilling 1984; Ormel \& Cuzzi 2007). This expression is valid for aggregates with stopping times $t_{\text {stop }}$ much smaller than $t_{\text {small. }}$ The velocity and turnover time of the largest eddies, $u_{\text {large }}$ and $t_{\text {large }}$, are related to $u_{\text {small }}$ and $t_{\text {small }}$ by $u_{\text {large }}=\operatorname{Re}^{1 / 4} u_{\text {small }}$ and $t_{\text {large }} \approx \operatorname{Re}^{1 / 2} t_{\text {small }}$, where $\operatorname{Re}=\nu_{\text {turb }} / \nu_{\text {mol }}$ is the Reynolds number. The molecular viscosity $\nu_{\mathrm{mol}}$ is written as $\nu_{\mathrm{mol}}=0.5 u_{\mathrm{g}} / n_{\mathrm{g}} \sigma_{\mathrm{mol}}$, where $\sigma_{\text {mol }}=2 \times 10^{-15} \mathrm{~cm}^{2}$ is the molecular collision cross section (Chapman \& Cowling 1970). We express the turbulence viscosity $\nu_{\text {turb }}=u_{\text {large }}^{2} t_{\text {large }}$ as $\nu_{\text {turb }}=\alpha_{\text {turb }} c_{s}^{2} \Omega_{\mathrm{K}}^{-1}$, where $\alpha_{\text {turb }}$ is the $\alpha$-parameter. The turnover time of the largest eddies is taken to be $t_{\text {large }} \approx \Omega_{\mathrm{K}}^{-1}$, and thus their velocity is given by $u_{\text {large }} \approx \sqrt{\alpha_{\text {turb }}} c_{s}$. We consider $\alpha_{\text {turb }}=0,10^{-4}, 10^{-3}$, and $10^{-2}$ in this study. Again, we replace $\Delta t_{\text {stop }}$ in equation (57) with $t_{\text {stop. }}$.

If both of colliding aggregates have the mean charge $\langle Z\rangle$, the electrostatic energy (40) is written as

$$
E_{\mathrm{el}} \approx \frac{\langle Z\rangle^{2} e^{2}}{2 a}=\frac{\Gamma^{2} a}{2 \lambda^{2}} \text {. }
$$

where $\Gamma$ is the master parameter defined in equation (23). Equation (58) overestimates a true repulsion energy when one (or both) of the aggregates has a positive charge $Z>0$, or a negative charge $-Z>0$ smaller than the average value $-\langle Z\rangle$. To account for the dispersion of the charge distribution, we introduce the "three-sigma" electrostatic energy

$$
E_{\mathrm{el}, 3 \sigma} \equiv \frac{\langle Z\rangle\left(\langle Z\rangle+3\left\langle\Delta Z^{2}\right\rangle^{1 / 2}\right) e^{2}}{2 a},
$$

where the dispersion $\left\langle\Delta Z^{2}\right\rangle^{1 / 2}$ is calculated from equation (24). $E_{\mathrm{el}, 3 \sigma}$ represents the electrostatic energy between two aggregates with charges $\langle Z\rangle$ and $\langle Z\rangle+3\left\langle\Delta Z^{2}\right\rangle^{1 / 2}$. The probability that a collision involves the electrostatic energy larger than $E_{\mathrm{el}, 3 \sigma}$ is as small as "three sigma" $\left(\sim 10^{-3}\right)$. Note that $E_{\mathrm{el}, 3 \sigma}$ is always smaller than $E_{\mathrm{el}}$ since $\langle Z\rangle$ is always negative. Also, $E_{\mathrm{el}, 3 \sigma}$ becomes negative if $-\langle Z\rangle$ is smaller than $3\left\langle\Delta Z^{2}\right\rangle^{1 / 2}$. This actually happens when the aggregate size is sufficiently small (see fig. 3a below).

We compute the charge state at each evolutionary stage from equation (34) with $\bar{a} \approx a(N), \bar{\sigma} \approx \sigma(N)$. The sticking coefficients are estimated by phonon theory to be $s_{\mathrm{i}} \approx 1$ and $s_{\mathrm{e}}=0.1 \ldots 1$ (Umebayashi 1983). We adopt $s_{\mathrm{i}}=1$ and $s_{\mathrm{e}}=0.3$ in this study. The results obtained in this section are insensitive to the choice of $s_{\mathrm{e}}$ as long as $0.1 \lesssim s_{\mathrm{e}} \lesssim 1$. We do not use the original equation (30) because the gas-phase ionization is negligible in the present case. The gas-phase recombination rate is typically $\beta \sim 10^{-12 \ldots-7} \mathrm{~cm}^{3} / \mathrm{s}$. Using this value, we can 
estimate $g(\Gamma)$ as

$$
\begin{aligned}
g(\Gamma) & \sim \frac{\beta \zeta n_{\mathrm{g}}}{u_{\mathrm{i}} s_{\mathrm{e}} u_{\mathrm{e}}\left(\sigma n_{\mathrm{d}}\right)^{2}} \\
& \sim 10^{-11 \ldots-6}\left(\frac{f_{\mathrm{dg}}}{10^{-2}}\right)^{-2}\left(\frac{r}{5 \mathrm{AU}}\right)^{3}\left(\frac{T}{130 \mathrm{~K}}\right)^{-1 / 2}\left(\frac{\zeta}{10^{-17} / \mathrm{s}}\right),
\end{aligned}
$$

independently of $N$. Therefore, equation (34) is valid unless dust is depleted and $f_{\text {dg }}$ decreases by many orders of magnitude.

To confirm that our semianalytical calculation does work well, we have also performed fully numerical calculations including multi-component ions. In the numerical calculations, a simple reaction model by Umebayashi \& Nakano (1980) is adopted. This reaction model involves five light ions $\left(\mathrm{H}^{+}, \mathrm{H}_{2}^{+}, \mathrm{H}_{3}^{+}, \mathrm{He}^{+}, \mathrm{C}^{+}\right)$, heavy molecular ions $\left(\mathrm{m}^{+}\right)$, metal ions $\left(\mathrm{M}^{+}\right)$, free electrons, and charged dust aggregates. Heavy molecular ions and metal ions are represented by $\mathrm{HCO}^{+}$and $\mathrm{Mg}^{+}$, respectively. We adopt the same values of the rate coefficients $\beta^{(k)}, \beta^{\prime}(k, l)$ and the neutral gas abundances $n_{\mathrm{g}}^{(k, l)} / n_{\mathrm{g}}$ as those used by Sano et al. (2000). As seen below, the dominant ions are metal ions, which is essentially due to the fast charge transfer from heavy molecules to metal atoms. With this fact, we set the average ion mass $m_{\mathrm{i}}$ to be the mass of $\mathrm{Mg}^{+}\left(m_{\mathrm{i}}=24 m_{\mathrm{H}}\right)$ in semianalytical calculations. The numerical solutions are obtained from equations (1)-(5). We remark that the full numerical calculation is far more time-consuming than the semianalytical one.

\subsection{Results}

\subsubsection{The fiducial case: $\alpha_{\mathrm{turb}}=0, a_{0}=0.1 \mu \mathrm{m}$}

Here we show the result for the case $\alpha_{\text {turb }}=0$ (i.e., laminar disk) and $a_{0}=0.1 \mu \mathrm{m}$ as a fiducial example. The results for different values of $a_{0}$ and $\alpha_{\text {turb }}$ are examined in $\$ 3.2 .2$ and $\$ 3.2 .3$, respectively.

Figure 3 shows the evolution of dust charge state and gas ionization state at $r=5 \mathrm{AU}, z=H$. Here each evolutionary stage is labeled by the number of constituent monomers in an aggregate, $N$. The evolution of the mean $\langle Z\rangle$ and dispersion $\left\langle\Delta Z^{2}\right\rangle$ of the dust charge distribution as well as the master parameter $\Gamma$ is shown in figure $3 a$. We find that $\Gamma$ increases with $N$ and reaches to the maximum value $\Gamma_{\max }=2.8$ at $N \approx 10^{7}$. This means that the gas-dust mixture is an iondust plasma $\left(\Theta \ll \Gamma_{\max }\right)$ at the initial stage, and evolves into an ion-electron plasma $\left(\Theta \gg \Gamma_{\max }\right)$ as the dust grows. This is expected from the analysis in the last section: in the BCCA growth, $\Theta \propto 1 /\left(\sigma a n_{\mathrm{d}}^{2}\right)$ is proportional to $N^{1 / 2}$, and thus increases with the growth. We have confirmed that the above value of $\Gamma_{\max }$ is consistent with the solution to equation (35).

It is useful to introduce the critical monomer number $N \equiv$ $N_{\text {max }}$ at which the transition from the ion-dust plasma regime to the ion-electron plasma regime occurs. As explained in $\S 2.3$, this value can be estimated by setting $\Theta \approx \Gamma_{\max }$, or

$$
\sigma a n_{\mathrm{d}}^{2} \approx \frac{\zeta n_{\mathrm{g}} \lambda}{u_{\mathrm{i}} \Gamma_{\max }} .
$$

Substituting $n_{\mathrm{d}} \approx n_{\mathrm{d} 0} / N, \sigma \approx \sigma_{0} N$, and $a \approx a_{0} N^{1 / 2}$ into this equation, we obtain the critical size for the transition

$$
N_{\max } \approx\left(\frac{\sigma_{0} a_{0} u_{\mathrm{i}} n_{\mathrm{d} 0}^{2} \Gamma_{\max }}{\zeta n_{\mathrm{g}} e^{2}}\right)^{1 / 2}
$$

$$
\begin{aligned}
\approx & 10^{6} f_{\Sigma}^{2}\left(\frac{f_{\mathrm{dg}}}{0.014}\right)^{4}\left(\frac{r}{5 \mathrm{AU}}\right)^{-6}\left(\frac{T}{130 \mathrm{~K}}\right)^{2}\left(\frac{\zeta}{10^{-17} / \mathrm{s}}\right)^{-2} \\
& \times\left(\frac{a_{0}}{0.1 \mu \mathrm{m}}\right)^{-6}\left(\frac{\rho_{0}}{1.4 \mathrm{~g} / \mathrm{cm}^{3}}\right)^{-4}
\end{aligned}
$$

or equivalently,

$$
\begin{aligned}
a_{\max } \approx & a_{0} N_{\max }^{1 / 2} \\
\approx & 10^{-2} f_{\Sigma}\left(\frac{f_{\mathrm{dg}}}{0.014}\right)^{2}\left(\frac{r}{5 \mathrm{AU}}\right)^{-3}\left(\frac{T}{130 \mathrm{~K}}\right)\left(\frac{\zeta}{10^{-17} / \mathrm{s}}\right)^{-1 / 2} \\
& \times\left(\frac{a_{0}}{0.1 \mu \mathrm{m}}\right)^{-2}\left(\frac{\rho_{0}}{1.4 \mathrm{~g} / \mathrm{cm}^{3}}\right)^{-2} \mathrm{~cm},
\end{aligned}
$$

where we have explicitly expressed the dependence on $f_{\Sigma}$.

The mean (negative) charge $-\langle Z\rangle$ is proportional to $N$ in the ion-dust regime $\left(N \ll N_{\max }\right)$, and is proportional to $N^{1 / 2}$ in the ion-electron regime $\left(N \gg N_{\max }\right)$. This is easily understood if one recalls that $-\langle Z\rangle \propto \Gamma a \propto \Gamma N^{1 / 2}$. For $N \ll N_{\max }$, $\Gamma \approx \Theta$ is proportional to $N^{1 / 2}$, so $-\langle Z\rangle \propto N$. For $N \gg N_{\max }$, $\Gamma$ approaches a constant, and thus $-\langle Z\rangle \propto N^{1 / 2}$. On the other hand, the dispersion $\left\langle\Delta Z^{2}\right\rangle^{1 / 2}$ is found to be nearly proportional to $N^{1 / 4}$, which is because $\left\langle\Delta Z^{2}\right\rangle^{1 / 2} \sim(a / \lambda)^{1 / 2}$ and $a \propto N^{1 / 2}$. It is important to notice here that the relative width $\left|\left\langle\Delta Z^{2}\right\rangle^{1 / 2} /\langle Z\rangle\right|$ of the charge distribution becomes sharper and sharper as the dust grows.

The transition of the plasma state is better illustrated by figure $3 \mathrm{~b}$. This figure shows the number densities of ions and electrons, $n_{\mathrm{i}}$ and $n_{\mathrm{e}}$, as well as the net dust charge density $\langle Z\rangle n_{\mathrm{d}}$. For $N \ll N_{\max }$, dust is the dominant carrier of negative charges, as expected for the ion-dust plasma state. The free electron density $n_{\mathrm{e}}$ is smaller than that of ions by a factor of $\left(1 / s_{\mathrm{e}}\right)\left(m_{\mathrm{e}} / m_{\mathrm{i}}\right)^{1 / 2} \sim 10^{-2}$ (see eq. [38]). As the aggregates grow and $N$ reaches the critical number $N_{\max }$, the dust charge density $-\langle Z\rangle n_{\mathrm{d}}$ begins to decrease and $n_{\mathrm{e}}$ begins to increase. Finally, at $N \approx 10^{7}$, free electrons become the dominant negative charge carrier, and the ion-electron plasma state $\left(n_{\mathrm{i}} \approx n_{\mathrm{e}}\right)$ is established.

Interestingly, the abundances of charged species, $n_{\mathrm{i}}$ and $n_{\mathrm{e}}$, are nearly constant for both limits of ion-dust and ion-electron plasma regimes. This result is in contrast to that of previous studies based on the classical, compact $(D=3)$ growth model (e.g., Sano et al. 2000; Wardle 2007) in which $n_{\mathrm{i}}$ and $n_{\mathrm{e}}$ increase as the dust grows. This difference is attributed to the fact that the net projected area $\sigma n_{\mathrm{d}}$ of dust aggregates is kept nearly constant for $D \lesssim 2$, while it decreases for $D=3$. Using the constancy $\sigma n_{\mathrm{d}}=\sigma_{0} n_{\mathrm{d} 0}$, equations (32) and (33) can be rewritten as $n_{\mathrm{i}}=n_{\mathrm{i} 0} /(1+\Gamma)$ and $n_{\mathrm{e}}=n_{\mathrm{i} 0}\left(s_{\mathrm{e}} u_{\mathrm{e}} / u_{\mathrm{i}}\right) \exp \Gamma$, where

$$
\begin{aligned}
n_{\mathrm{i} 0}= & \frac{\zeta n_{\mathrm{g}}}{u_{\mathrm{i}} \sigma_{0} n_{\mathrm{d} 0}} \\
\approx & 10^{-14}\left(\frac{f_{\mathrm{dg}}}{0.014}\right)^{-1}\left(\frac{r}{5 \mathrm{AU}}\right)^{3}\left(\frac{\zeta}{10^{-17} / \mathrm{s}}\right) \\
& \times\left(\frac{a_{0}}{0.1 \mu \mathrm{m}}\right)^{-3}\left(\frac{\rho_{0}}{1.4 \mathrm{~g} / \mathrm{cm}^{3}}\right)^{-1} n_{\mathrm{g}}
\end{aligned}
$$

is the abundance of ions for $N \ll N_{\max }$. For both plasma limits, the factors $1+\Gamma$ and $\exp \Gamma$ are approximately constant, so both $n_{\mathrm{i}}$ and $n_{\mathrm{e}}$ approach constant values. Physically speaking, the constancy of $\sigma n_{\mathrm{d}}$ for $D \lesssim 2$ means that all the monomers in a fractal aggregate with $\widetilde{D} \lesssim 2$ are exposed to outer space, and are thus capable to capture free electrons and ions.

Figure 3 also shows the result of full numerical calculations using the simplified ion-reaction scheme. We find an excellent agreement between the semianalytical and full numerical 

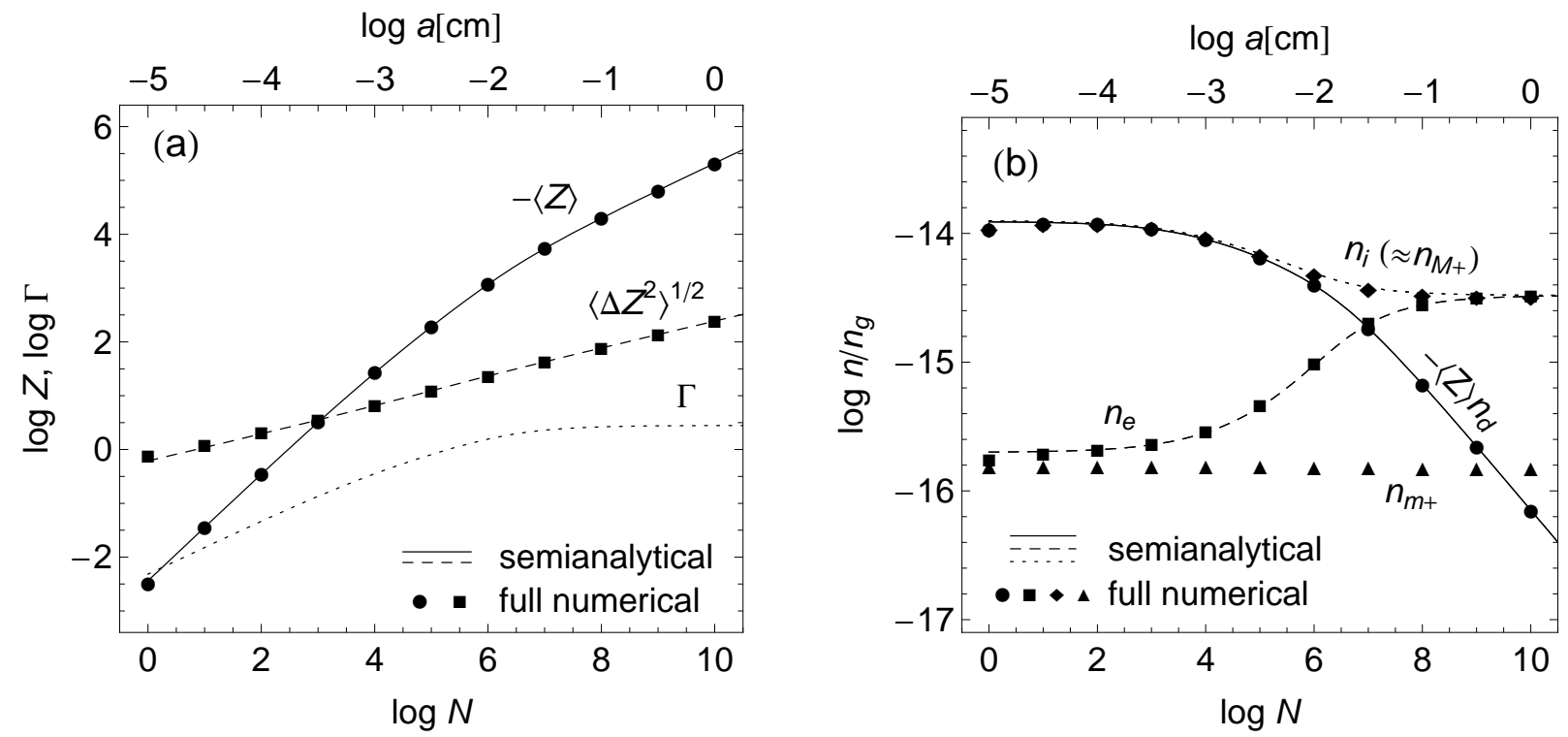

FIG. 3.- (a) The evolution of the dust charge distribution for the "BCCA" (i.e., quasi-monodisperse, $D \approx 2$ ) dust growth. The solid, dashed, and dotted curves represent the average $\langle Z\rangle$, dispersion $\left\langle\Delta Z^{2}\right\rangle^{1 / 2}$, and master parameter $\Gamma$ of the charge distribution calculated using the semianalytical method (eqs. [23], [24], and [34]). The filled circles and squares denote $\langle Z\rangle$ and $\left\langle\Delta Z^{2}\right\rangle^{1 / 2}$ obtained from full-numerical calculations using the Umebayashi \& Nakano model (see $\S 3.1 .3$ ). (b) The evolution of the gas ionization state and the dust charge state for the BCCA growth. $n_{\mathrm{i}}$ and $n_{\mathrm{e}}$ are the number densities of ions and electrons in the gas phase, and $\langle Z\rangle n_{\mathrm{d}}$ is the net dust charge density. $n_{\mathrm{M}^{+}}$and $n_{\mathrm{m}^{+}}$are the number densities of metal ions and molecular ions.

$\log a[\mathrm{~cm}]$

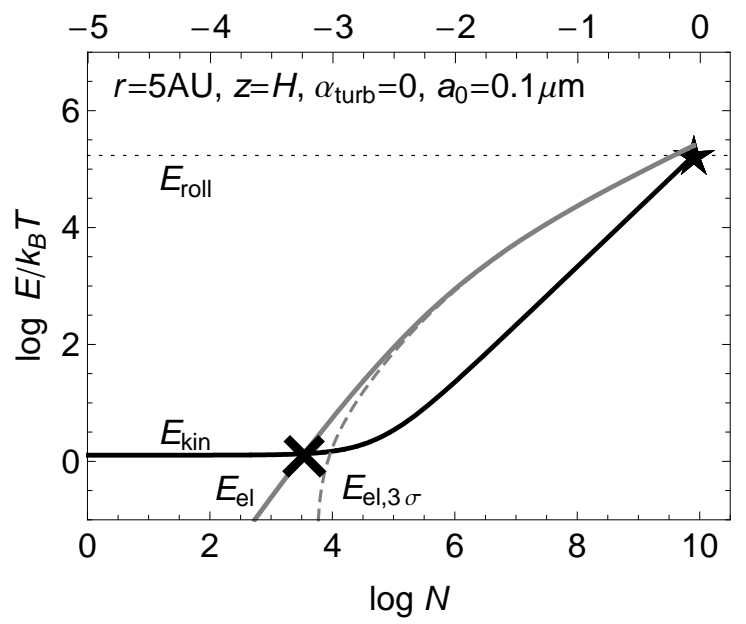

FIG. 4.- The relative kinetic energy $E_{\text {kin }}$ (eq. [52]; solid black curve) versus the electrostatic energy $E_{\text {el }}$ (eq. [ [58] ]; solid gray curve) for the BCCA growth as a function of the monomer number $N$. The disk position is $(r, z)=(5 \mathrm{AU}, H)$, and the turbulence parameter and the monomer size are set to $\alpha_{\text {turb }}=0$ (i.e., laminar) and $a_{0}=0.1 \mu \mathrm{m}$. The collision cross section for an aggregate pair with $E_{\mathrm{el}}$ and $E_{\text {kin }}$ vanishes when $E_{\mathrm{el}}>E_{\mathrm{kin}}$ (see eq. [42]), meaning that the BCCA growth "freezes out" at the size indicated by the cross $(\times)$ symbol. The dashed gray curve shows the "three-sigma" electrostatic energy $E_{\mathrm{el}, 3 \sigma}$ (eq. [59), representing the effect of charge fluctuation. The star $(\star)$ symbol indicates the size at which $E_{\text {kin }}$ reaches the critical rolling-friction energy $E_{\text {roll }}$ (eq. [50]; dotted black curve). Above this critical energy, collisional compression of aggregates becomes effective.

calculations. It is clear that our semianalytical approach is not only efficient but also accurate. The most abundant ions are metal ions for all stages of dust evolution. Molecular ions, the second most abundant ones, are an order of magnitude fewer than metal ions.

Now we examine the growth condition. Figure 4 shows the kinetic energy $E_{\text {kin }}$ and electrostatic energy $E_{\text {el }}$ for colliding aggregates at each evolutionary stages. For $N \lesssim 10^{5}(a \lesssim$ $30 \mu \mathrm{m}$ ), the thermal (Brownian) motion dominates the rela- tive velocity of the aggregates, so $E_{\mathrm{kin}}$ is kept constant $\approx k_{\mathrm{B}} T$. Fore $N \gtrsim 10^{5}$, the vertical sedimentation dominates the aggregate motion and $E_{\text {kin }}$ increases with $N$. On the other hand, $E_{\text {el }}$ always grows with $N$, in proportional to $N^{3 / 2}$ for $N \lesssim N_{\max }$ and to $N^{1 / 2}$ for $N \gtrsim N_{\max }$. This is explained from the fact that $|\langle Z\rangle| \propto N$ for the ion-dust plasma regime $\left(N \lesssim N_{\max }\right)$ and $|\langle Z\rangle| \propto a \propto N^{1 / 2}$ for the ion-electron plasma regime $\left(N \gtrsim N_{\max }\right)$. As a result, the growth condition (42) breaks down at $N \approx 10^{3.5}(a \approx 6 \mu \mathrm{m})$. This means that the collision between aggregates with average charge $\langle Z\rangle$ becomes impossible at this stage. Note that the repulsion energy $E_{\mathrm{el}}$ reaches 10 times the thermal energy $\sim k_{\mathrm{B}} T$ as early as $N \approx 10^{4}$, and finally goes up to $10^{5} k_{\mathrm{B}} T$ at the onset of collisional compression. It is evident that the thermal fluctuation of the kinetic energy cannot help the aggregates to grow beyond $N \gg 10^{4}$.

One may expect that the fluctuation of aggregate charge could help the growth. To see this effect, we overplot in figure 4 the "three-sigma" electrostatic energy $E_{\mathrm{el}, 3 \sigma}$ defined in equation (59). We see that $E_{\mathrm{el}, 3 \sigma}$ quickly converges to $E_{\text {el }}$ and finally exceeds $E_{\text {kin }}$ at $N \approx 10^{4}$. This is expected from figure 3 3 : the relative width $\left|\langle Z\rangle /\left\langle\Delta Z^{2}\right\rangle^{1 / 2}\right|$ of the the charge distribution becomes narrower and narrower as the dust grows. Therefore, the result that the aggregates cannot grow beyond $N \gg 10^{4}$ is preserved even if the charge fluctuation is taken into account. ${ }^{2}$ All the above facts suggest that the BCCA growth of dust aggregates at this disk position "freezes out" at size $N \sim 10^{4}(a \sim 10 \mu \mathrm{m})$.

It is interesting to compare this critical size for the freezeout with that for the first compression, i.e., the size at which the growth mode changes from the BCCA to the growth involving collisional compression. We overplot the critical

${ }^{2}$ It is also found that $E_{\mathrm{el}, 3 \sigma}$ is negative for smaller sizes, $N \lesssim 10^{3.7}$. This means that colliding aggregates can possess opposite charges with a probability larger than "three-sigma". This is because the average negative charge $-\langle Z\rangle$ in this stage is smaller than $3\left\langle\Delta Z^{2}\right\rangle^{1 / 2}$, as seen in figure $3 a$. However, the attraction energy $-E_{\mathrm{el}, 3 \sigma}$ is insignificant: at most half of the kinetic energy $E_{\text {kin }}$. 
rolling energy $E_{\text {roll }}$ (eq. [50]) in figure 4. Comparing this critical energy with $E_{\text {kin }}$, we find that the collisional compression begins at size $N \approx 10^{10}(a \approx 1 \mathrm{~cm})$, i.e., many orders of magnitude smaller than the above critical freeze-out size. This illustrates how fast the charging of aggregates begins to affect their collisional growth.

Figure 5] compares the energy ratio $E_{\mathrm{el}} / E_{\text {kin }}$ for different disk positions. We find that the barrier against the BCCA growth appears irrespectively of the disk radius $r$. By contrast, the growth barrier vanishes at $z \gtrsim 3 H$ because the sedimentation velocity at the high altitudes is large enough for aggregates to overcome the barrier.

To summarize, the freeze-out of the BCCA growth is very likely to occur in this fiducial model, except at high altitudes over the midplane.

As seen in figure 4, the energy ratio $E_{\mathrm{el}} / E_{\text {kin }}$ takes its maximum at $N \approx N_{\max }$. With this fact, we can roughly estimate the maximum value $\left(E_{\mathrm{el}} / E_{\mathrm{kin}}\right)_{\max }$ of the energy ratio at $z \approx H$ as follows. We assume that the relative motion of aggregates by $N \approx N_{\max }$ is dominated by vertical sedimentation, as is for $r=5 \mathrm{AU}$. Then, substituting $\Delta u \approx \Delta u_{\text {sed }}$ into equation (52) and using equations (58) and (62), we obtain

$$
\begin{aligned}
\left(\frac{E_{\mathrm{el}}}{E_{\mathrm{kin}}}\right)_{\max } \approx & 30 f_{\Sigma}\left(\frac{f_{\mathrm{dg}}}{0.014}\right)^{-2}\left(\frac{\zeta}{10^{-17} / \mathrm{s}}\right) \\
& \times\left(\frac{a_{0}}{0.1 \mu \mathrm{m}}\right)^{-1}\left(\frac{\rho_{0}}{1.4 \mathrm{~g} / \mathrm{cm}^{3}}\right)^{-1}
\end{aligned}
$$

at $z \approx H$. Notably, $\left(E_{\mathrm{el}} / E_{\mathrm{kin}}\right)_{\max }$ is explicitly independent of both $r$ and $T$. This means that the "height" of the growth barrier is insensitive to the temperature profile. Equation (65) does not hold beyond $r \approx 5 \mathrm{AU}$, since the maximum energy ratio appears in the Brownian motion regime. This equation is nevertheless useful because it allows us a rough estimation on how the growth barrier depend on the model parameters. For example, for fixed $\zeta, a_{0}$, and $\rho_{0}$, the the growth barrier is more serious if the disk is more massive $\left(f_{\Sigma}>1\right)$ or more depleted of dust $\left(f_{\mathrm{dg}}<10^{-2}\right)$.

\subsubsection{Effect of monomer size}

The actual size of dust monomers in protoplanetary disks is unknown. Infrared observations of interstellar medium suggest the size distribution of interstellar grains ranges from $\approx 0.005 \mu \mathrm{m}$ to $\approx 0.25 \mu \mathrm{m}$ (MRN distribution; Mathis et al. 1977). If interstellar grains are not aggregates but monomers, the typical monomer size in protoplanetary disks will fall within the range $0.001 \mu \mathrm{m} \lesssim a \lesssim 1 \mu \mathrm{m}$. Figure 6 compares the energy ratio $E_{\text {el }} / E_{\text {kin }}$ for different monomer sizes $a_{0}=$ $0.01,0.1$, and $1 \mu \mathrm{m}$. We find that $N_{\max } \propto a_{0}^{-6}\left(a_{\max } \propto a_{0}^{-2}\right)$ and $\left(E_{\mathrm{el}} / E_{\mathrm{kin}}\right)_{\max } \propto a_{0}^{-1}$, as expected from equations (62) and (65). This delay (measured in the "degree of growth" $N$ ) is attributed to the larger cross section of BCCA clusters composed of smaller monomers. Such a larger cross section causes a quick depletion of free electrons in the gas phase, resulting in the delay of transition from the ion-dust plasma regime to the ion-electron regime. In addition, the larger cross section produces a stronger coupling to the gas, and in turn a slower increase of the kinetic energy. We find that the growth condition (42) breaks down much before the onset of collisional compression for all $a_{0} \lesssim 1 \mu \mathrm{m}$. Therefore, the "freeze-out" of the BCCA growth is not prevented even if the monomer size in protoplanetary disks is assumed to the maximum value inferred by the MRN distribution.

\subsubsection{Effect of turbulence}

Here we examine how strong turbulence is needed to remove the growth barrier. Figure 7 shows the evolution of energy ratio $E_{\text {el }} / E_{\text {kin }}$ for three turbulent cases $\alpha_{\text {turb }}=10^{-4}, 10^{-3}$, and $10^{-2}$. We see that turbulence of $\alpha_{\text {turb }} \lesssim 10^{-4}$ does not affect the energy ratio for any size $N$. This is not surprising because both $\Delta u_{\text {turb }}$ and $\Delta u_{\text {sed }}$ scale with $\Delta t_{\text {stop. }}$. The relative velocity $\Delta u_{\text {turb }}$ induced by turbulence is estimated as $\Delta u_{\text {turb }} \approx \Delta t_{\text {stop }} \operatorname{Re}^{1 / 4} u_{\text {large }} / t_{\text {large }} \sim \Delta t_{\text {stop }} \operatorname{Re}^{1 / 4} \alpha_{\text {turb }}^{1 / 2} \Omega_{\mathrm{K}}^{2} H$. At $z \sim H$, the Reynolds number Re is of order $\sim \alpha_{\mathrm{turb}} \Sigma_{\mathrm{g}} \sigma_{\mathrm{mol}} / m_{\mathrm{g}}$, so $\Delta u_{\text {turb }}$ is written as

$$
\Delta u_{\text {turb }} \sim\left(\frac{\Sigma_{\mathrm{g}} \sigma_{\mathrm{mol}}}{m_{\mathrm{g}}}\right)^{1 / 4} \alpha_{\text {turb }}^{3 / 4} \Delta t_{\text {stop }} \Omega_{\mathrm{K}}^{2} H
$$

On the other hand, the relative velocity by differential sedimentation is $\Delta u_{\text {sed }} \sim \Delta t_{\text {stop }} \Omega_{\mathrm{K}}^{2} H$, so we find

$$
\frac{\Delta u_{\text {turb }}}{\Delta u_{\text {sed }}} \sim\left(\frac{\Sigma_{\mathrm{g}} \sigma_{\mathrm{mol}}}{m_{\mathrm{g}}}\right)^{1 / 4} \alpha_{\mathrm{turb}}^{3 / 4} \sim\left(\frac{\alpha_{\text {turb }}}{10^{-4}}\right)^{3 / 4}\left(\frac{r}{5 \mathrm{AU}}\right)^{-3 / 8} .
$$

Therefore, the effect of turbulence on the aggregate collision is negligible for all $N$ as long as $\alpha_{\text {turb }} \lesssim 10^{-4}$.

For $\alpha_{\text {turb }} \gtrsim 10^{-2}$, we find that the growth barrier is entirely removed. This suggests that relatively strong $\left(\alpha_{\text {turb }} \gtrsim 10^{-2}\right)$ turbulence is a key ingredient for early stages of dust coagulation. In $\S 4.3$, we discuss this topic in more detail.

\section{DISCUSSION}

\subsection{Validity of the charge equilibrium}

In this study, we have assumed that the charge reactions are much faster than the dust-dust collisions. Now we show that this assumption is actually valid for evolutionary stages which we are interested in.

The typical time scale for the system to relax to an ionization-recombination equilibrium can be measured by the average time $t_{\text {coll, }}$ needed for an aggregate to collide with an ion,

$$
t_{\mathrm{coll}, \mathrm{i}}^{-1} \approx u_{\mathrm{i}} \sigma_{\mathrm{di}} n_{\mathrm{i}} \approx \frac{\zeta n_{\mathrm{g}}}{n_{\mathrm{d}}},
$$

where we have used that $\zeta n_{\mathrm{g}} \approx u_{\mathrm{i}} \sigma_{\mathrm{di}} n_{\mathrm{i}} n_{\mathrm{d}}$ since the gasphase recombination is negligible in the presence of dust (see $\S 3.1 .3$ ). On the other hand, the mean collision time $t_{\text {coll, }}$ between aggregates is written as

$$
t_{\text {coll, } \mathrm{d}}^{-1} \approx \sigma_{\mathrm{dd}} n_{\mathrm{d}} \Delta u \sim \sigma n_{\mathrm{d}} \Delta u .
$$

Therefore, the ratio of these time scales is estimated as

$$
\frac{t_{\text {coll }, \mathrm{d}}}{t_{\text {coll }, \mathrm{i}}} \sim \frac{\zeta n_{\mathrm{g}}}{\sigma n_{\mathrm{d}}^{2} \Delta u} \sim f_{\mathrm{dg}}^{-2}\left(\frac{m}{m_{\mathrm{g}}}\right)^{2} \frac{c_{s}}{\Delta u} \frac{m_{\mathrm{g}}}{\sigma \Sigma_{\mathrm{g}}} \frac{\zeta}{\Omega_{\mathrm{K}}} .
$$

If the dust velocity is dominated by the Brownian motion, as is for small aggregates, $\Delta u \sim c_{s} \sqrt{m_{\mathrm{g}} / m}$ and

$$
\begin{aligned}
\left.\frac{t_{\text {coll, }}}{t_{\text {coll }, \mathrm{i}}}\right|_{\text {Brown }} & \sim f_{\text {dg }}^{-2}\left(\frac{m}{m_{\mathrm{g}}}\right)^{5 / 2} \frac{m_{\mathrm{g}}}{\sigma \Sigma_{\mathrm{g}}} \frac{\zeta}{\Omega_{\mathrm{K}}} \\
& \sim N^{3 / 2}\left(\frac{f_{\mathrm{dg}}}{10^{-2}}\right)^{-2}\left(\frac{\zeta}{10^{-17} / \mathrm{s}}\right)\left(\frac{r}{2 \mathrm{AU}}\right)^{3},
\end{aligned}
$$

where we have used $a_{0} \sim 0.1 \mu \mathrm{m}$ and $\rho_{0} \sim 1 \mathrm{~g} / \mathrm{cm}^{3}$. We find that dust coagulation can be safely neglected if $r \gtrsim 2 \mathrm{AU}$ 
$\log a[\mathrm{~cm}]$

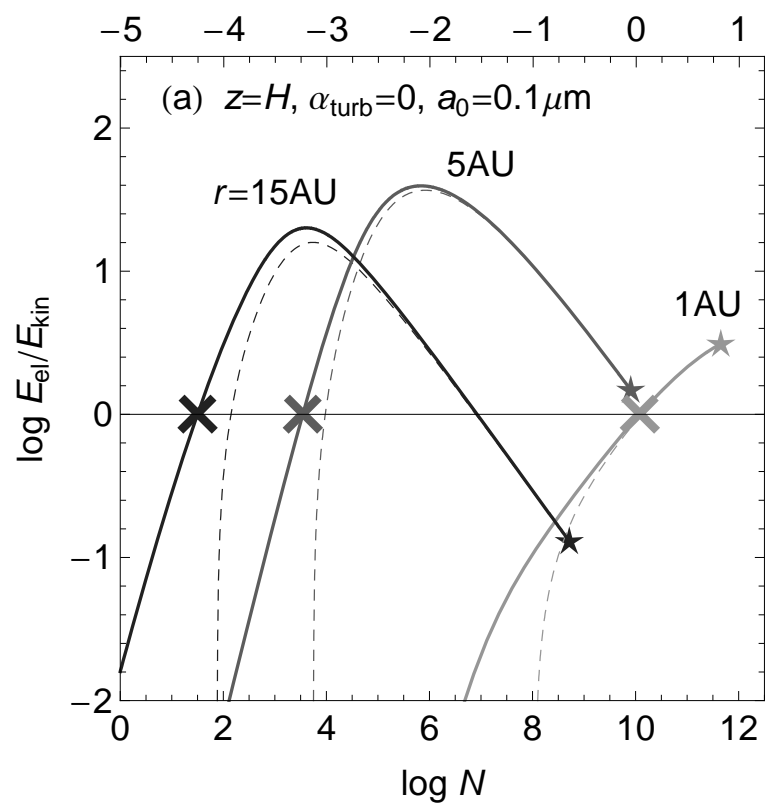

$\log a[\mathrm{~cm}]$

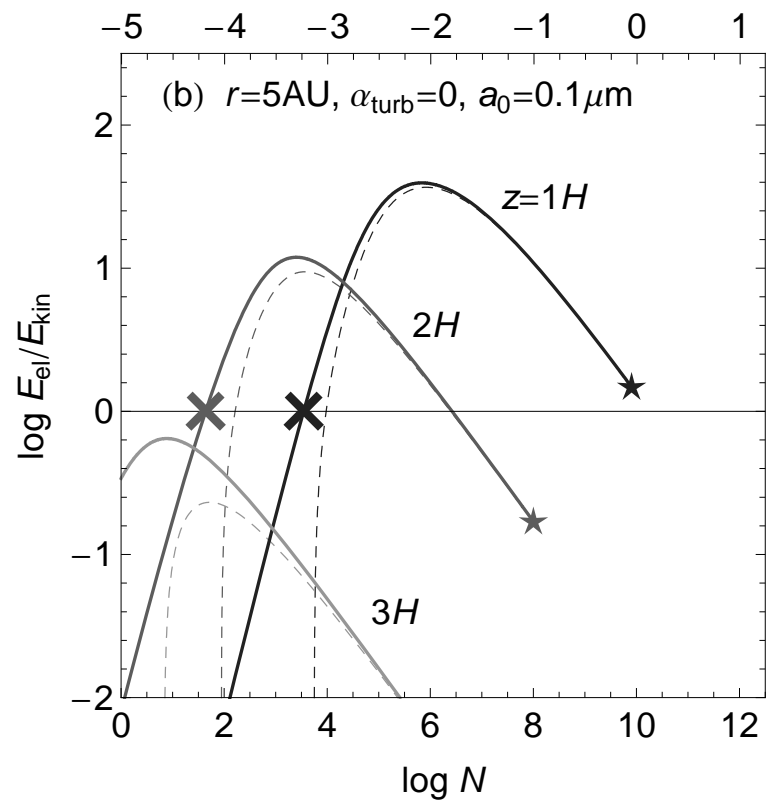

FIG. 5.- The ratios $E_{\mathrm{el}} / E_{\mathrm{kin}}$ between the electrostatic and kinetic energies (solid curves) at various disk radii $r(a)$ and altitudes $z(b)$ as functions of $N$. The $\alpha$-parameter and the monomer radius are set to $\alpha_{\text {turb }}=0$ and $a_{0}=0.1 \mu \mathrm{m}$. The cross $(\times)$ symbols indicate the size at which the growth condition 42 breaks down for aggregates with mean charge $\langle Z\rangle$. The dashed curves show the ratio $E_{\mathrm{el}, 3 \sigma} / E_{\mathrm{kin}}$ for the "three-sigma" energy, which represents the effect of charge dispersion. The star $(\star)$ symbols indicate the sizes at which $E_{\text {kin }}$ reaches the critical rolling-friction energy $E_{\text {roll }}$.

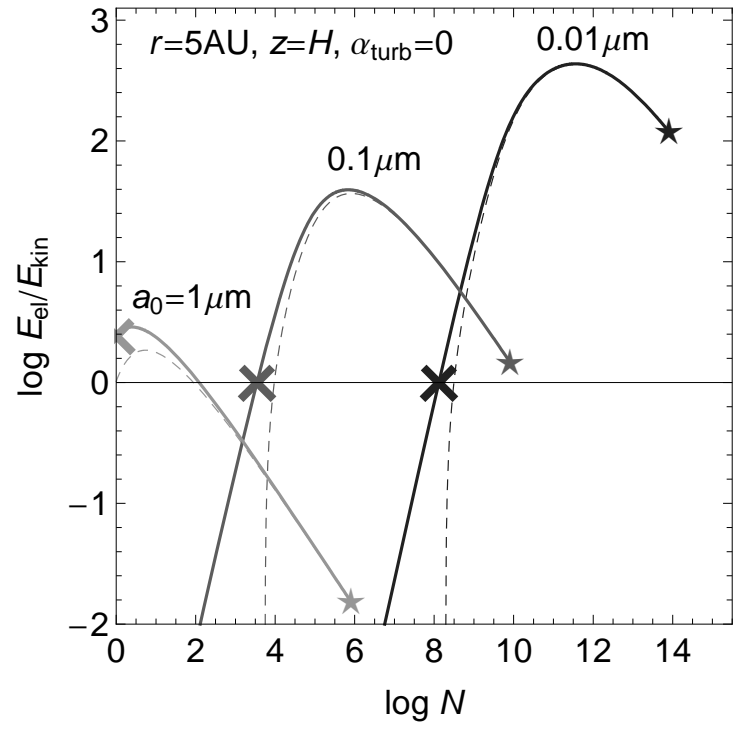

FIG. 6.- The energy ratios $E_{\mathrm{el}} / E_{\mathrm{kin}}$ (solid curves) at $r=5 \mathrm{AU}$ and $z=H$ for different monomer sizes $a_{0}$. The cross $(\times)$ symbols indicate the "freezeout" sizes at which the growth condition (42) breaks down. The dashed curves show $E_{\mathrm{el}, 3 \sigma} / E_{\mathrm{kin}}$. The star $(\star)$ symbols indicate the sizes at which $E_{\text {kin }}$ reaches the critical rolling-friction energy $E_{\text {roll }}$.

or $N \gg 1$. This is true even if the motion of dust aggregates is dominated by vertical sedimentation, since $\Delta u_{\text {sed }} \sim$ $c_{s}\left(m / \Sigma_{\mathrm{g}} \sigma\right)$, and thus

$$
\begin{aligned}
\left.\frac{t_{\mathrm{coll}, \mathrm{d}}}{t_{\mathrm{coll}, \mathrm{i}}}\right|_{\mathrm{sed}} & \sim f_{\mathrm{dg}}^{-2} \frac{m}{m_{\mathrm{g}}} \frac{\zeta}{\Omega_{\mathrm{K}}} \\
& \sim 10^{3} N\left(\frac{f_{\mathrm{dg}}}{10^{-2}}\right)^{-2}\left(\frac{\zeta}{10^{-17} / \mathrm{s}}\right)\left(\frac{r}{2 \mathrm{AU}}\right)^{3 / 2} .
\end{aligned}
$$

Thus, it is concluded that the growth of dust aggregates can be neglected if $r \gtrsim 2 \mathrm{AU}$ or $N \gg 1$.

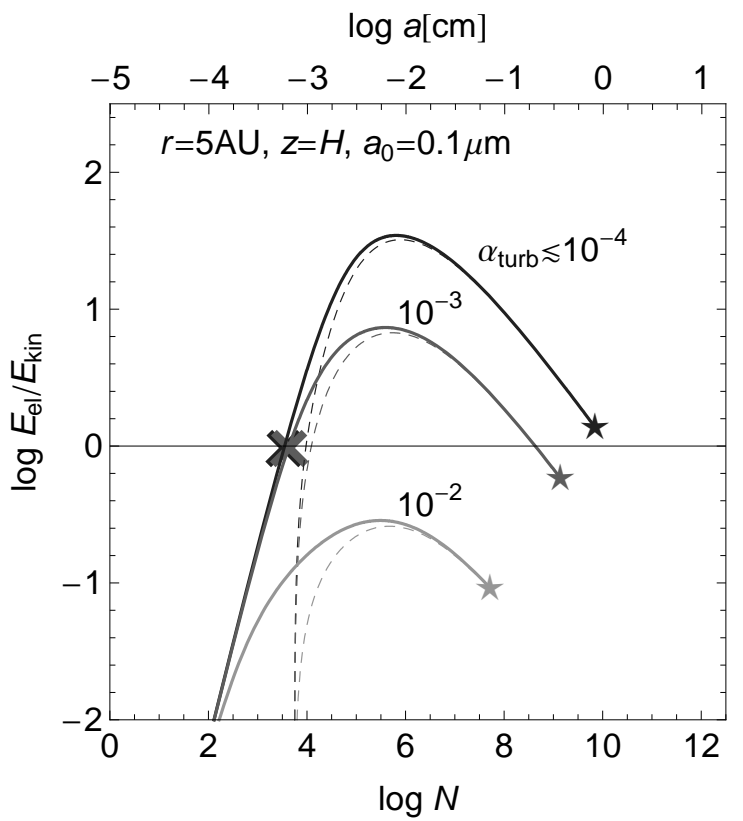

FIG. 7.- The energy ratios $E_{\mathrm{el}} / E_{\mathrm{kin}}$ (solid curves) at $r=5 \mathrm{AU}$ and $z=$ $H$ for different turbulence strengths $\alpha_{\text {turb }}$. The cross $(\times)$ symbols indicate the "freeze-out" sizes at which the growth condition (42) breaks down. The dashed curves show $E_{\mathrm{el}, 3 \sigma} / E_{\mathrm{kin}}$. The star $(\star)$ symbols indicate the sizes at which $E_{\text {kin }}$ reaches the critical rolling-friction energy $E_{\text {roll }}$.

\subsection{Internal electrostatic force}

In the last section, we have implicitly assumed that charged aggregates can stick to each other as long as the collision condition (42) is satisfied. One might wonder if the collided aggregates are pulled off from each other by the electrostatic repulsion. In fact, this repulsion is much weaker than the attraction (due to van der Waals force) between two monomers in contact. The electrostatic repulsion force $F_{\mathrm{el}, \text { int }}$ acting be- 
tween two collided aggregates is estimated as

$$
F_{\text {el,int }} \sim \frac{(Z e)^{2}}{a^{2}} \sim \frac{\Gamma^{2} e^{2}}{\lambda^{2}} \lesssim 10^{-8}\left(\frac{T}{130 \mathrm{~K}}\right)^{2} \mathrm{dyn},
$$

where we have used that $\Gamma<\Gamma_{\max } \sim 3$. Note that maximum value of $F_{\mathrm{el}, \text { int }}$ is independent of the aggregate size a. On the other hand, the critical force needed to separate two monomers in contact is (Johnson et al. 1971; Dominik \& Tielens 1997)

$$
F_{\text {crit }}=3 \pi \gamma \frac{a_{0}}{2} \sim 10^{-3}\left(\frac{\gamma}{10^{2} \mathrm{~g} / \mathrm{cm}^{2}}\right)\left(\frac{a_{0}}{0.1 \mu \mathrm{m}}\right) \text { dyn, }
$$

where $\gamma$ is the surface adhesive energy mentioned in the last section. Thus, the electrostatic force inside an aggregate is negligibly weak compared to the contact force between two constituent monomers.

\subsection{Dust growth in strong turbulence}

As seen in $\$ 3.2 .3$, the electrostatic barrier against the fractal growth will be removed if considerably strong $\left(\alpha_{\text {turb }} \gtrsim 10^{-2}\right)$ turbulence is present. We here discuss whether such turbulence is likely to occur in protoplanetary disks, and what would happen after the dust overcome the electrostatic growth barrier.

The most robust mechanism for disk turbulence is MRI ${ }^{3}$. MRI-driven turbulence will achieve $\alpha_{\text {turb }} \sim 10^{-2}$ in its saturated state (e.g. Sano et al. 1998). Therefore, fractal aggregates will be able to overcome the electric barrier in MRIactive regions. Sano et al. (2000) calculated the active region using the MMSN model and found that MRI will be active only at outer $(r \gtrsim 20 \mathrm{AU})$ disk radii or high $(|z| \gtrsim 2 H)$ altitudes if the dust size is $0.1 \mu \mathrm{m}$. The size of the active region does not vary even if the dust grows since, as seen in $\$ 3.2 .1$, the ionization fraction is kept nearly constant as long as the dust undergoes the fractal growth. Therefore, the region in which the fractal aggregates can overcome the electrostatic barrier is limited to outer disk radii and high altitudes.

A more serious problem is that such strong turbulence causes another kind of growth barrier, i.e., catastrophic fragmentation of colliding aggregates. In turbulent regions, large aggregates with $t_{\text {stop }} \sim 1 / \Omega_{\mathrm{K}}$ have the maximum collisional velocity of order $u_{\text {large }} \sim \sqrt{\alpha_{\text {turb }}} c_{s}$, which amounts to more than $100 \mathrm{~m} / \mathrm{s}$ for $\alpha_{\text {turb }} \gtrsim 10^{-2}$. On the other hand, as shown by recent $N$-body simulations (Wada et al.2008), catastrophic fragmentation will take place for relative velocity $\Delta u \gtrsim$ $30 \mathrm{~m} / \mathrm{s}$. Therefore, it is very likely that strong turbulence destroys the aggregates and consequently prevents further dust growth. This idea is supported by a recent statistical study (Brauer et al. 2008).

Thus, the combination of electric repulsion and collisional fragmentation might strictly limit the dust growth and subsequent planetesimal formation in protoplanetary disks. It is important to think of a possibility that dust evolution will continue in some way even if the turbulence is weak and the quasi-monodisperse fractal growth does freeze out. This is the topic of the next subsection.

\subsection{A possible scenario to overcome the electric growth barrier}

\footnotetext{
${ }^{3}$ It is unknown whether any mechanism other than MRI can drive and sustain turbulence with $\alpha_{\text {turb }} \gtrsim 10^{-2}$ in the early stage of dust evolution. For example, convective instability may operate in this stage (Lin \& Papaloizou 1980 ), but it is unlikely to sustain such strong turbulence (Stone \& Balbus 1996).
}

In $\S 3$, we have ignored the size distribution of aggregates. In fact, there may exist some aggregates considerably larger than average-sized ones. In the following, we consider whether such large aggregates can continue to grow even if the growth of average-sized ones has frozen out.

Let us consider a small population of irregularly large aggregates (referred to as "test aggregates") growing with a large population of standard $(D \sim 2)$ fractal aggregates ("field aggregates"). Under this assumption, the kinetic energy of relative motion between test and field aggregates is written as

$$
E_{\mathrm{kin}, \mathrm{tf}}=\frac{1}{2} \frac{m_{\mathrm{t}} m_{\mathrm{f}}}{m_{\mathrm{t}}+m_{\mathrm{f}}}\left(\Delta u_{\mathrm{tf}}\right)^{2} \approx \frac{1}{2} m_{\mathrm{f}}\left(\Delta u_{\mathrm{tf}}\right)^{2}=\frac{1}{2}\left(\frac{\Delta u_{\mathrm{tf}}}{\Delta u_{\mathrm{ff}}}\right)^{2} E_{\mathrm{kin}, \mathrm{ff}},
$$

where the subscripts ' $t$ ' and ' $\mathrm{f}$ ' respectively represent the test and field aggregates, and we have used the assumption $m_{\mathrm{t}} \gg$ $m_{\mathrm{f}} . E_{\mathrm{kin}, \mathrm{ff}}$ and $\Delta u_{\mathrm{ff}}$ are the kinetic energy of relative motion and the relative velocity between two field aggregates, and are thus equivalent to $E_{\text {kin }}$ and $\Delta u$ in $\S 3$. On the other hand, the electrostatic energy between test and field aggregates is

$$
E_{\mathrm{el}, \mathrm{tf}}=\frac{a_{\mathrm{t}} a_{\mathrm{f}} \Gamma^{2} e^{2}}{a_{\mathrm{t}}+a_{\mathrm{f}}} \approx \frac{\Gamma^{2} a_{\mathrm{f}}}{\lambda^{2}}=\frac{1}{2} E_{\mathrm{el}, \mathrm{ff}},
$$

where $E_{\mathrm{el}, \mathrm{ff}}$ is equivalent to $E_{\mathrm{el}}$ in $\S 3$, and we have used that $a_{\mathrm{t}} \gg a_{\mathrm{f}}$. Thus, the energy ratio $E_{\mathrm{el}, \mathrm{ff}} / E_{\mathrm{kin}, \mathrm{ff}}$ is written as

$$
\frac{E_{\mathrm{el}, \mathrm{ft}}}{E_{\mathrm{kin}, \mathrm{ft}}} \approx \frac{E_{\mathrm{el}, \mathrm{ff}}}{E_{\mathrm{kin}, \mathrm{ff}}}\left(\frac{\Delta u_{\mathrm{ff}}}{\Delta u_{\mathrm{tf}}}\right)^{2} .
$$

Now we assume that the growth of field aggregates has frozen out due to the electric barrier, i.e., $E_{\mathrm{kin} . f f}=E_{\mathrm{el}, \mathrm{ff}}$. At this stage, the condition for the collision between test and field aggregates, $E_{\text {kin,tf }}>E_{\text {el,tf }}$, reduces to a simple inequality

$$
\Delta u_{\mathrm{tf}}>\Delta u_{\mathrm{ff}}
$$

We readily notice that Brownian motion does not satisfy this condition since $\Delta u_{\mathrm{tf}} \approx \sqrt{8 k_{\mathrm{B}} T / \pi m_{\mathrm{f}}}$ and $\Delta u_{\mathrm{ff}} \approx$ $\sqrt{16 k_{\mathrm{B}} T / \pi m_{\mathrm{f}}}$. The remained possibilities are the differential sedimentation and turbulent-driven motion. In both cases, the relative velocity $\Delta u$ is proportional to $\Delta t_{\text {stop }}$, or $\Delta(\mathrm{m} / \sigma)$. It is very important to notice here that the condition (78) is not safely satisfied as long as the test aggregate is as fluffy as field aggregates, i.e., $m_{\mathrm{t}} / \sigma_{\mathrm{t}}$ is comparable to $m_{\mathrm{f}} / \sigma_{\mathrm{f}}$. Hence, the condition (78) will be safely satisfied only if the test aggregate is more compact and has larger $m / \sigma$ than the field aggregates. Moreover, collision with a smaller aggregate generally tends to increase $m_{\mathrm{t}} / \sigma_{\mathrm{t}}$, allowing the test aggregate the next collision. Therefore, if there exists an aggregate that is large and compact, it will be able to continue growing by sweeping up smaller "frozen" aggregates.

The above consideration suggests that the freeze-out of the quasi-monodisperse fractal growth may not mean the termination of dust evolution. Rather, it may be the beginning of bimodal growth in which only a small fraction of aggregates can grow larger and larger while the rest remain frozen. We plan to examine this possibility in more detail in the future studies. In any case, we expect that the effect of dust charging should qualitatively modify the current scenario of dust growth in protoplanetary disks.

\section{SUMMARY}

In this study, we have investigated the electric charging of dust aggregates and its effect on collisional dust growth in protoplanetary disks. We have found that the conditions for 
ionization-recombination equilibrium are reduced to a single equation (eq. [30]). Just by solving this equation numerically, the dust charge state and gas ionization state can be analytically computed for an arbitrary ensemble of aggregates in a self-consistent way. It is also confirmed that our semianalytical method reproduces the results of a previously used, more complicated numerical method (\$3.2.1, fig.3). This formalism thus provides a fast charge-state solver that will allow a coupled simulation of MRI-driven turbulence and dust coagulation.

As an application, we have explored the effect of electrostatic charging on an early stage of dust coagulation in protoplanetary disks. We considered the quasi-monodisperse fractal growth with the fractal dimension $D \sim 2$ as suggested by previous laboratory experiments and $N$-body simulations (Blum 2004; Dominik et al. 2007). Our findings are summarized as follows:

1. For a wide range of model parameters, the effective cross section for the mutual collision of aggregates is quickly suppressed as the fractal growth proceeds and finally vanishes at a certain aggregate size $(\$ \$ 3.2 .1,3.2 .2)$. This is due to the strong electrostatic repulsion between aggregates charging negatively on average, and happens much before the collisional compression of aggregates becomes effective. Both the charge fluctuation and the thermal velocity fluctuation do not help the aggregates to overcome the growth barrier. Without strong turbulence, the quasi-monodisperse fractal growth is very likely to "freeze out" on its way to the subsequent growth stage.

2. Strong $\left(\alpha_{\text {turb }} \gtrsim 10^{-2}\right)$ turbulence will help the aggregates to overcome the above growth barrier (\$3.2.3). However, such turbulence is likely to occur only in MRI-active regions, i.e., at outer disk radii or high altitudes (§4.3). Furthermore, it will cause another serious problem - the catastrophic disruption of collided aggregates-in later stages. These facts suggest that the combination of electric repulsion and collisional disruption may strictly limit the collisional growth of dust aggregates in protoplanetary disks.

3. The freeze-out of the fractal growth might be followed by bimodal growth in which only a small fraction of large aggregates can continue growing while a large fraction of small fractal aggregates remains frozen (\$4.4). This could qualitatively change the current scenario of planetesimal formation in protoplanetary disks (Dominik et al. 2007). We will examine this possibility in more detail in forthcoming papers.

Finally, we point out that the fractal $(D \lesssim 2)$ dust growth tends to keep the ionization degree of the disk small due to the open nature of aggregates (\$3.2.1, fig. 3b). This means that the magnetorotationally unstable region hardly expands until the collisional compression of the aggregates begins to work. This conclusion is in contrast to that of previous studies (e.g., Sano et al. 2000; Wardle 2007) which claimed that the ionization degree increase as the aggregates grow. However, they assumed compact dust growth, which clearly contradicts recent laboratory experiments and $N$-body simulations. Thus, the magnetorotational stability of protoplanetary disks must be reexamined taking into account that the fractal nature of dust aggregates.

The author thanks M. Sakagami, S. Inutsuka, and H. Tanaka for careful reading of the manuscript and for valuable comments.

\section{APPENDIX}

\section{DERIVATION OF THE CHARGE DISTRIBUTION}

Since the velocity of free electrons is much greater than that of ions, dust aggregates charge up negatively on average. Thus, let us assume $Z<0$ and use the expressions of $\sigma_{\mathrm{di}}$ and $\sigma_{\mathrm{de}}$ valid for $Z<0$. Then, equation (18) is written as

$$
s_{\mathrm{i}} u_{\mathrm{i}} n_{\mathrm{i}}\left(1-\frac{Z}{\tau}\right) n_{\mathrm{d}}(I, Z)=s_{\mathrm{e}} u_{\mathrm{e}} n_{\mathrm{e}} \exp \left(\frac{Z+1}{\tau}\right) n_{\mathrm{d}}(I, Z+1),
$$

where $\tau=a / \lambda$.

We make the following assumption:

$$
\frac{1}{\left\langle\Delta Z^{2}\right\rangle^{1 / 2}} \sim \frac{\left\langle\Delta Z^{2}\right\rangle^{1 / 2}}{\tau} \sim \varepsilon,
$$

where $\left\langle\Delta Z^{2}\right\rangle$ is the variance of the charge state distribution, and $\varepsilon \ll 1$. We also assume that $n_{\mathrm{d}}(I, Z)$ varies with a typical scale $\sim\left\langle\Delta Z^{2}\right\rangle^{1 / 2}$. Under these assumptions, $n_{\mathrm{d}}(I, Z+1)$ can be written as

$$
n_{\mathrm{d}}(I, Z+1)=n_{\mathrm{d}}(I, Z)+\frac{\partial n_{\mathrm{d}}}{\partial Z}(I, Z)+O\left(\varepsilon^{2}\right) .
$$

Also, $\exp [(Z+1) / \tau]$ is written as $\exp (Z / \tau)+O\left(\varepsilon^{2}\right)$ since $\tau^{-1}=\left\langle\Delta Z^{2}\right\rangle^{-1 / 2} \cdot\left\langle\Delta Z^{2}\right\rangle^{1 / 2} / \tau \sim \varepsilon^{2}$. Substituting them into equation (A1), we obtain a first-order differential equation for $n_{\mathrm{d}}(I, Z)$,

$$
\frac{\partial n_{\mathrm{d}}}{\partial Z}(I, Z)+W(I, Z) n_{\mathrm{d}}(I, Z) \approx 0
$$

where

$$
W(I, Z) \equiv 1-\frac{s_{\mathrm{i}} u_{\mathrm{i}} n_{\mathrm{i}}(1-Z / \tau)}{s_{\mathrm{e}} u_{\mathrm{e}} n_{\mathrm{e}} \exp (Z / \tau)} .
$$

Equation $\mathrm{A4}$ is accurate to terms of the first order in $\varepsilon$.

Let us denote the solution of $W(I, Z)=0$ by $Z_{0}$ and write $Z=Z_{0}+\delta Z$. Also, we make an additional approximation that $|\delta Z| \ll \tau$. Expanding $W(I, Z)$ in powers of $|\delta Z| / \tau$ and using $W\left(I, Z_{0}\right)=0$, we have

$$
W(I, Z) \approx \frac{2-Z_{0} / \tau}{1-Z_{0} / \tau} \frac{\delta Z}{\tau}
$$


which is accurate to the first order in $|\delta Z| / \tau$. Hence, equation (A4) is approximated by

$$
\frac{\partial n_{\mathrm{d}}}{\partial Z}(I, Z)+\frac{\delta Z}{\left\langle\Delta Z^{2}\right\rangle} n_{\mathrm{d}}(I, Z) \approx 0,
$$

where

$$
\left\langle\Delta Z^{2}\right\rangle \equiv \frac{1-Z_{0} / \tau}{2-Z_{0} / \tau} \tau
$$

It is an easy task to show that the solution to (A7) is a Gaussian distribution (22) with average $\langle Z\rangle=Z_{0}$ and variance $\left\langle\Delta Z^{2}\right\rangle$. Rewriting $Z_{0}$ and $\tau$ using $Z_{0}=-\Gamma \tau$ and $\tau=a / \lambda$, we obtain equations (23) and (24). We note that equation (A8) has been also obtained by Draine \& Sutin (1987), but they did not show the derivation of this equation in their paper.

Now we justify the assumption (A2). The first part of the assumption $\left\langle\Delta Z^{2}\right\rangle^{-1 / 2} \sim\left\langle\Delta Z^{2}\right\rangle^{1 / 2} / \tau$, which is equivalent to $\left\langle\Delta Z^{2}\right\rangle \sim$ $\tau$, is always satisfied since $\tau / 2<\left\langle\Delta Z^{2}\right\rangle<\tau$ (see eq. [A8]). The second part $\left\langle\Delta Z^{2}\right\rangle^{1 / 2} / \tau \sim \varepsilon \ll 1$ is also satisfied in typical protoplanetary disks if dust aggregates are not as small as constituent monomers $(\sim 0.1 \mu \mathrm{m})$, because

$$
\frac{\left\langle\Delta Z^{2}\right\rangle^{1 / 2}}{\tau} \sim \tau^{-1 / 2} \sim\left(\frac{a}{0.1 \mu \mathrm{m}}\right)^{-1 / 2}\left(\frac{T}{130 \mathrm{~K}}\right)^{-1 / 2} .
$$

It is noted that the approximation $|\delta Z| \ll \tau$ used to obtain equation (A6) is rewritten as $|\delta Z| \ll \tau^{1 / 2}\langle\Delta Z\rangle^{1 / 2}$. Hence, this approximation is good as long as the region $|\delta Z| \lesssim\langle\Delta Z\rangle^{1 / 2}$ is considered.

\section{REFERENCES}

Anders, E., \& Grevesse, N. 1989, Geochim. Cosmochim. Acta, 53, 197

Balbus, S. A., \& Hawley, J. F. 1991, ApJ, 376, 214

Blum, J.,Wurm, G., Poppe, T., \& Heim, L.-O. 1998, Earth Moon Planets, 80, 285

Blum, J., et al. 2000, Phys. Rev. Lett., 85, 2426

Blum, J. 2004, in ASP Conf. Ser. 309, Astrophysics of Dust, ed. A. N. Witt, G.C. Clayton, \& B. T. Draine (San Francisco: ASP), 369

Brauer, F., Dullemond, C. P., Henning, Th. 2008, A\&A, 480, 859

Chapman, S., \& Cowling, T. G. 1970, The Mathematical Theory of Nonuniform Gases (London: Cambridge Univ. Press)

Dominik, C. P., Blum, J., Cuzzi, J. N., \& Wurm, G. 2007, in Protostars and Planets V, ed. B. Reipurth, D. Jewitt, \& K. Keil (Tucson: Univ. Arizona Press), 783

Dominik, C., \& Tielens, A. G. G. M. 1997, ApJ, 480, 647

Draine, B. T., \& Sutin, B. 1987, ApJ, 320, 803

Gammie, C. F. 1996, ApJ, 457, 355

Glassgold, A. E., Najita, J., \& Igea, J. 1997 ApJ, 480, 344

Hayashi, C. 1981, Prog. Theor. Phys. Suppl., 70, 35

Heim, L.-O., Blum, J., Preuss, M., \& Butt, H.-J. 1999, Phys. Rev. Lett., 83, 3328

Igea, J. \& Glassgold, A. E. 1999, ApJ, 518, 848

Ilgner, M., \& Nelson, R. P. 2006a, A\&A, 445, 205

Ilgner, M., \& Nelson, R. P. 2006b, A\&A, 445, 223

Ilgner, M., \& Nelson, R. P. 2006c, A\&A, 445, 731

Inutsuka, S. \& Sano, T. 2005, ApJ, 628, L155

Israelachvili, J. 1992, Intermolecular and Surface Forces (2nd ed.; London: Academic Press)

Ivlev, A. V., Morfill, G. E., \& Konopka, U. 2002, Phys. Rev. Lett., 89, 195502 Jackson, J. D. 1998, Classical Electrodynamics (3rd ed.; New York: Wiley)

Johnson K. L., Kendall, K., \& Roberts, A. D. 1971, Proc. R. Soc. London A, 324, 301

Kempf, S., Pfalzner, S., \& Henning, T. K. 1999, Icarus, 141, 388

Konopka, U., Mokler F., Ivlev, A. V., Kretschmer, M., Morfill, G. E., et al. 2005, New Journal of Physics, 7, 227

Lin, D. N. C., \& Papaloizou, J. C. B. 1980, MNRAS, 191, 37
Mathis, J.S., Rumpl, W., \& Nordsieck, K. H. 1977, ApJ, 217, 425

Meakin, P., \& Donn, B. 1988, ApJ, 329, L39

Meakin, P., Donn, B., \& Mulholland, G. W. 1989, Langmuir, 5, 510

Meakin, P. 1991, Rev. Geophys., 29, 317

Minato, T., Köhler, M., Kimura, H., Mann, I., and Yamamoto, T. 2006, A\&A, 452,701

Nakagawa, Y., Nakazawa, K., \& Hayashi, C. 1981, Icarus, 45, 517

Ormel, C. W., \& Cuzzi, J. N. 2007, A\&A, 466, 413

Pollack, J. B., Hollenbach, D., Beckwith, S., Simonelli, D. P., Roush, T., \& Fong, W. 1994, ApJ, 421, 615

Sano, T., Inutsuka, S., \& Miyama, S. M. 1998, ApJ, 506, L57

Sano, T., Miyama, S. M., Umebayashi, T. \& Nakano, T. 2000, ApJ, 543, 486

Shukla, P. K., \& Mamun, A. A. 2002, Introduction to Dusty Plasma Physics (Bristol: IoP)

Spitzer, L. 1941, ApJ, 93, 369.

Stone, J. M., \& Balbus, S. A. 1996, ApJ, 464, 364

Suyama, T., Wada, K., \& Tanaka, H. 2008, ApJ, 684, 1310

Tanaka, H., Himeno Y., \& Ida, S. 2005, ApJ, 625, 414

Turner, N. J., \& Sano, T. 2008, ApJ, 679, L131

Umebayashi, T. 1983, Prog. Theor. Phys., 69, 480

Umebayashi, T., \& Nakano, T. 1980, PASJ, 32, 405

Umebayashi, T., \& Nakano, T. 1981, PASJ, 33, 617

Umebayashi, T.. \& Nakano, T. 1990, MNRAS, 243, 103

Umebayashi, T.. \& Nakano, T. 2009, ApJ, 690, 69

Wada, K., Tanaka, H., Suyama, T., Kimura, H., \& Yamamoto, T. 2008, ApJ, 677,1296

Wardle, M. 2007, Ap\&SS, 311, 35

Weidenschilling, S. J. 1980, Icarus, 44, 172

Weidenschilling, S. J. 1984, Icarus, 60, 553

Wolk, S. J., Harnden, F. R., Flaccomio, E., Micela, G., Favata, F., Shang, H., \& Feigelson, E. D. 2005, ApJ, 160, 423

Wurm, G., \& Blum, J. 1998, Icarus, 132, 125 